ARTICLE

DOI: $10.1038 /$ s41467-017-01600-6

\title{
STIM1 promotes migration, phagosomal maturation and antigen cross-presentation in dendritic cells
}

Paula Nunes-Hasler (10 ${ }^{1}$, Sophia Maschalidi ${ }^{2,3}$, Carla Lippens ${ }^{4}$, Cyril Castelbou ${ }^{1}$, Samuel Bouvet ${ }^{1}$ Daniele Guido ${ }^{1}$, Flavien Bermont ${ }^{1}$, Esen Y. Bassoy (10 ${ }^{1}$, Nicolas Page ${ }^{4}$, Doron Merkler ${ }^{4}{ }^{4}$, Stéphanie Hugues ${ }^{4}$, Denis Martinvalet ${ }^{1}$, Bénédicte Manoury ${ }^{3,6,7} \&$ Nicolas Demaurex (1) ${ }^{1}$

Antigen cross-presentation by dendritic cells (DC) stimulates cytotoxic T cell activation to promote immunity to intracellular pathogens, viruses and cancer. Phagocytosed antigens generate potent $\mathrm{T}$ cell responses, but the signalling and trafficking pathways regulating their cross-presentation are unclear. Here, we show that ablation of the store-operated-Ca ${ }^{2+}$-entry regulator STIM1 in mouse myeloid cells impairs cross-presentation and DC migration in vivo and in vitro. Stiml ablation reduces $\mathrm{Ca}^{2+}$ signals, cross-presentation, and chemotaxis in mouse bone-marrow-derived DCs without altering cell differentiation, maturation or phagocytic capacity. Phagosomal pH homoeostasis and ROS production are unaffected by STIM1 deficiency, but phagosomal proteolysis and leucyl aminopeptidase activity, IRAP recruitment, as well as fusion of phagosomes with endosomes and lysosomes are all impaired. These data suggest that STIM1-dependent $\mathrm{Ca}^{2+}$ signalling promotes the delivery of endolysosomal enzymes to phagosomes to enable efficient cross-presentation.

\footnotetext{
${ }^{1}$ Department of Cell Physiology and Metabolism, University of Geneva, Geneva 1211, Switzerland. ${ }^{2}$ Laboratory of Normal and Pathological Homeostasis of the Immune System, INSERM UMR1163, Paris 75015, France. ${ }^{3}$ Université Paris Descartes, Sorbonne Paris Cité, Faculté de médecine Paris Descartes, Paris 75015, France. ${ }^{4}$ Department of Pathology and Immunology, University of Geneva, Geneva 1211, Switzerland. ${ }^{5}$ Division of Clinical Pathology, Geneva University Hospital, Geneva 1211, Switzerland. ${ }^{6}$ Institut National de la Santé et de la Recherche Médicale, Unité 1151, Paris 75014, France. ${ }^{7}$ Centre National de la Recherche Scientifique, Unité 8253, Paris 75014, France. Correspondence and requests for materials should be addressed to P.N.-H. (email: Paula.Nunes@unige.ch)
} 
D endritic cells (DC) are phagocytic immune cells that link innate and adaptive immunity by processing and presenting ingested antigens. One of the unique functions of DCs is cross-presentation, which is a specific type of antigen presentation that occurs via major histocompatibility complex class I (MHC-I) molecules to activate $\mathrm{CD}^{+} \mathrm{T}$ cells and help generate antigen-specific immunity to intracellular pathogens, viruses and cancer cells. Cross-presentation of antigens acquired through phagocytosis produces more potent $\mathrm{T}$ cell responses than soluble antigens ${ }^{1}$, and DCs are particularly involved in phagocytosis and transport of large particles $(>500 \mathrm{~nm})$ to draining lymph nodes ${ }^{2}$. However, the precise molecular mechanisms by which cross-presentation of phagocytosed antigens occurs are not well understood. Cross-presentation requires a number of proteins normally located in the endoplasmic reticulum (ER), such as tapasin, calreticulin, ERp57 and the translocon Sec61 ${ }^{1}$. DC phagosomes are particularly rich in ER proteins ${ }^{3,4}$, but the signalling and trafficking mechanisms regulating the relationship between the ER and the phagosome during cross-presentation is controversial $3,5-8$

$\mathrm{Ca}^{2+}$ signalling is linked to a variety of DC functions including differentiation, maturation, migration, cytokine secretion, phagocytic ingestion and antigen presentation ${ }^{9}$. However, most studies have relied on the use of non-specific inhibitors, ionophores and chelators, which can have pleiotropic effects. Stromal interaction molecule (STIM) proteins, which include the two isoforms STIM1 and STIM2 each with multiple splice variants, are ER transmembrane proteins that sense the $\mathrm{ER} \mathrm{Ca}^{2+}$ depletion resulting from activation of inositol trisphosphate $\left(\mathrm{InsP}_{3}\right)$ receptors ${ }^{10}$. They subsequently remodel the ER and promote the formation and expansion of membrane contact sites (MCS) between the ER and plasma membrane (ER-PM MCS), where they directly activate PM-resident $\mathrm{Ca}^{2+}$ channels of the ORAI and transient receptor potential (TRPC) families in the process termed store-operated $\mathrm{Ca}^{2+}$-entry $(\mathrm{SOCE})^{11}$. Electrophysiological studies suggest that SOCE is the major $\mathrm{Ca}^{2+}$ entry pathway in $\mathrm{DCs}^{12}$, and one study suggests that STIM2 is the major isoform regulating DC function in mice ${ }^{13}$. In human peripheral blood monocyte-derived DCs genetic manipulation of ORAI1 and STIM1 suggested that STIM1 is critical for DC maturation ${ }^{14}$, but another study suggests that STIM1 and STIM2 are dispensable for a variety of DC functions in mice ${ }^{15}$.

Although the classic model of cross-presentation postulates that antigens are first partially proteolysed in phagosomes, retrotranslocated from the phagosome to the cytosol where they are further processed by the proteasome, and then reimported into the ER for loading onto ER-resident MHC-I molecules ${ }^{1}$, some studies propose that non-canonical trafficking pathways involving fusion of ERGIC vesicles and recycling endosomes with phagosomes may explain the presence of ER proteins on phagosomes ${ }^{7}$, 8,16 . However, the signalling and targeting mechanisms that control these pathways are unclear. In neutrophils, we previously showed that STIM1 promotes the formation of contact sites between the ER and phagosomes that allow localized $\mathrm{Ca}^{2+}$ signalling ${ }^{17}$, raising the question of whether STIM1 may also affect the association between phagosomes and the ER in DCs.

In the present study, we characterize the consequences of genetic ablation of Stim1 on DC functions including differentiation, maturation, migration, phagocytosis and cross-presentation. Our data establish that STIM1 is the major isoform controlling SOCE in mouse DCs and suggest that STIM1 promotes crosspresentation at least in part by increasing $\mathrm{Ca}^{2+}$-dependent migration. In addition, STIM1 promotes the formation of contact sites between the ER and phagosomes that in turn produce localized $\mathrm{Ca}^{2+}$ signals that may potentiate proteolysis and fusion of phagosomes with endosomes and lysosomes.

\section{Results}

Stim1 promotes cross-presentation of phagocytosed antigens. To determine whether STIM1 promotes cross-presentation, PBS solutions with $0,0.5$, or $1 \%$ ovalbumin (OVA)-coated beads (OVAb) were injected into footpads of LysM-Cre; Stim $1^{\text {flfl }}$ mice bearing the CD45.2 allele and a conditional deletion of the Stim1 gene in myeloid cells, and into control CD45.2 Stim $1^{f l / f l}$ littermates. After $24 \mathrm{~h}, \mathrm{CD} 45.1, \mathrm{H} 2-\mathrm{K}^{\mathrm{b}} / \mathrm{OVA}(257-264)$-reactive CD8 $\alpha$ $+\mathrm{T}$ cells (OT-I) labelled with carboxyfluorescein succinimidyl ester (CFSE) were injected retro-orbitally. Draining (DL) and non-draining (NDL) lymph nodes were harvested after $72 \mathrm{~h}$, and the total number of CD45.1 $1^{+}$OT-I cells within the CD8 $\alpha^{+}$ population, as well as the CFSE dilution as a measure of OT-I proliferation, were determined. The full gating strategy is shown in Fig. 1a. STIM1 deficiency dramatically reduced the total number of CD45.1 $1^{+}$OT-I cells within the CD8 $\alpha^{+}$gate in DL of mice injected with 1 or $0.5 \%$ OVAb but not in NDL (Fig. 1a, b) or in lymph nodes from mice injected with PBS (Supplementary Fig. 1a). OT-I proliferation was reduced in a dose-dependent manner (Fig. 1a, b), indicating that effectively, cross-presentation was impaired upon Stim1 ablation in myeloid cells.

We next examined whether cell-specific DC cross-presentation was impaired by co-culturing bone-marrow-derived DCs (BMDC) pulsed with OVAb along with OT-I cells in vitro and measuring OT-I proliferation after $72 \mathrm{~h}$ by bromodeoxyuridine (BrdU) incorporation. Western blots confirmed that STIM1 protein expression was reduced by $>90 \%$ in BMDCs isolated from LysM-Cre; Stim $1^{\text {flffl }}$ mice (labelled henceforth Stim $1^{-/-}$for simplicity) as compared to BMDCs isolated from Stim ${ }^{f l / f l}$ mice (labelled henceforth WT, Supplementary Fig. 1b). Maturation with microbial products $\mathrm{CpG}$ oligodeoxynucleotides and lipopolysaccharide (LPS) increased OT-I proliferation by $\sim$ twofold (Fig. 1c), while $\mathrm{Ca}^{2+}$ chelation with BAPTA-AM or inhibition of InsP $_{3}$-receptors with Xestospongin $\mathrm{C}$ (Xesto) decreased crosspresentation, as did the inhibitors of the NADPH oxidase and the vacuolar-ATPase, diphenylpropidium iodide (DPI) and concanamycin A (ConcA) respectively, applied as positive controls (Fig. 1d). This indicates that cross-presentation is $\mathrm{Ca}^{2+}$-dependent. Stim1 ablation reduced the relative levels of crosspresentation to similar extents in immature and mature cells, an effect that was most pronounced at intermediate DC:T cell ratios (Fig. 1c). Similar results were obtained when measuring OT-I proliferation by CFSE dilution (Supplementary Fig. 1c, d). The Stim1-dependent defect in cross-presentation was specific for processing of phagocytosed antigens, as STIM1-deficient BMDCs were equally efficient at cross-presenting as WT when pulsed with varying doses of SIINFEKL peptide, an OVA(257-264) fragment that does not require proteolytic processing (Fig. 1e, Supplementary Fig. 1e). To confirm that abrogating STIM1 expression reduces cross-presentation in vitro, a CD $8 \alpha^{+} \mathrm{DC}$ cell line highly efficient at cross-presentation, DC ${ }^{211418}$, was transduced with either control or shRNA directed against STIM1 (shCTR and shSTIM1 respectively). STIM1 depletion was verified by Western blot (Supplementary Fig. 1b). Similar to primary BMDCs, crosspresentation was reduced by STIM1 depletion in $\mathrm{DC}^{2114}$ cells (Fig. 1f). DC ${ }^{2114}$ induced nearly fourfold higher levels of OT-I proliferation as compared to BMDCs (Supplementary Fig. 1f), generating large colonies easily discerned by microscopy where the effect of STIM1 knockdown was clearly apparent (Supplementary Fig. 1g). These data indicate that STIM1 promotes the cross-presentation of phagocytosed antigens in vivo and that this effect is recapitulated in vitro in primary or cultured DCs.

Stim1 ablation impairs the $\mathrm{Ca}^{2+}$-dependent migration of BMDCs. The decrease in the total number of OT-I cells in the DL 
of STIM1-deficient mice, a measure of functional cross-presentation, could result from defective migration of DCs to and from the site of OVAb injection where antigens are captured, to the lymph nodes where DCs encounter OT-I cells. To test whether DC migration to lymph nodes was impaired in vivo, a 1:1 mixture of BMDCs from CD45.2 WT mice expressing cytosolic GFP (WT-GFP) and CD45.2 Stim1 ${ }^{-/-}$BMDCs was coinjected into footpads of CD45.1 host mice together with $0.5 \%$

a
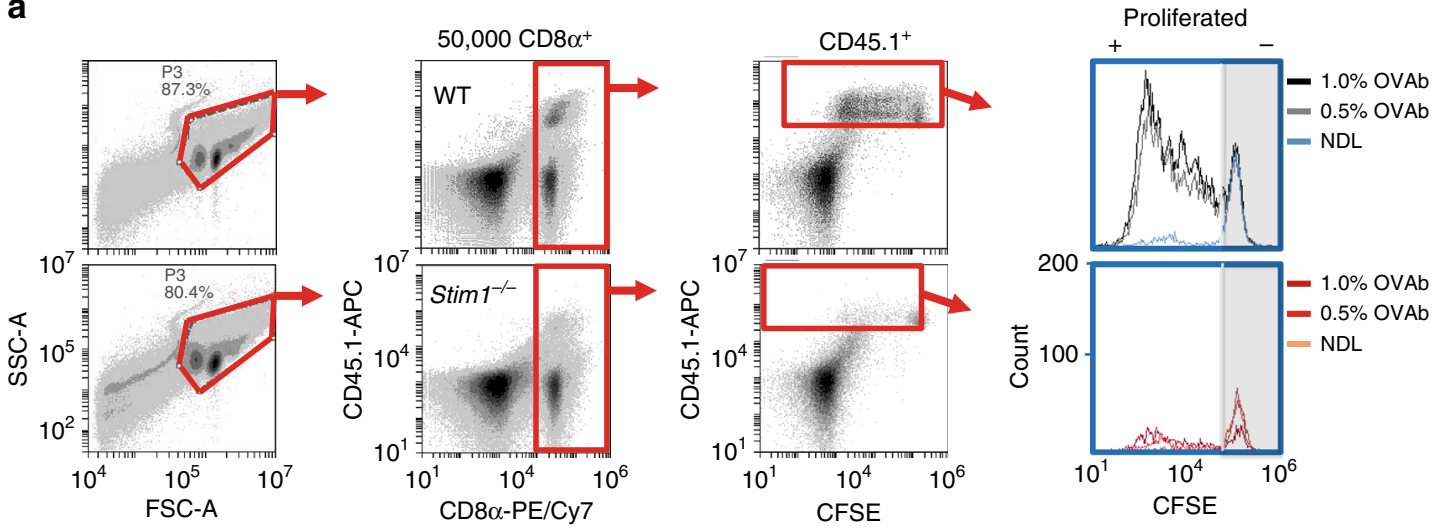

b
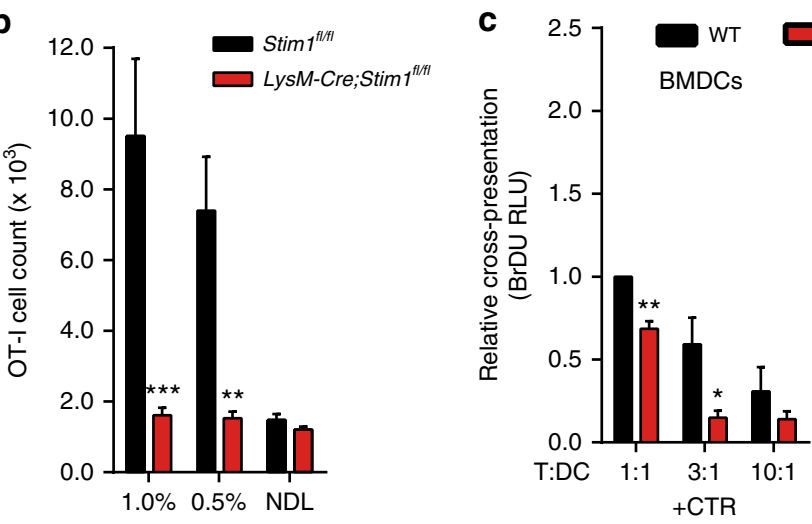

Stim $1^{-1-}$

$+\mathrm{OVAb}$
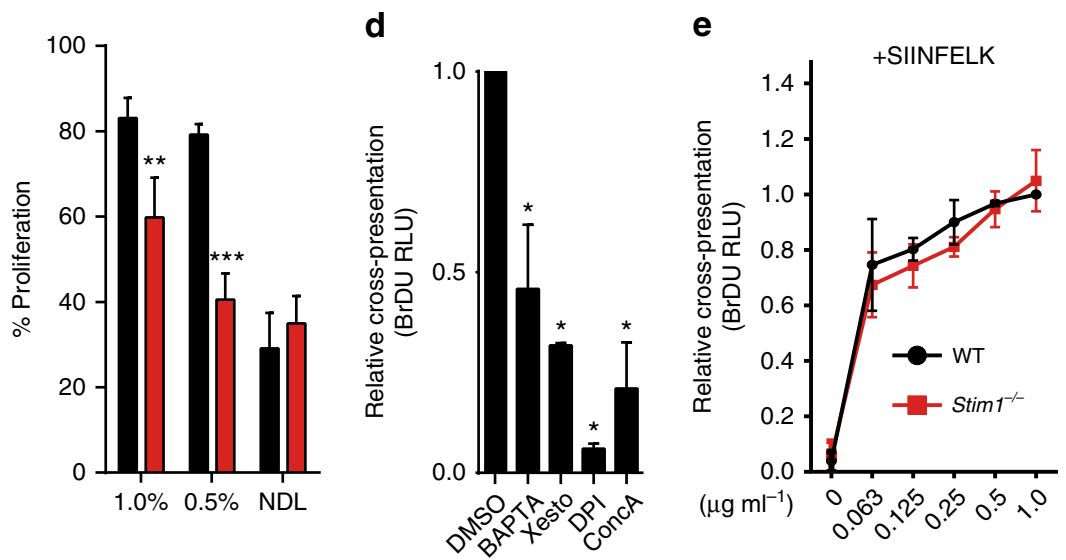

$f$
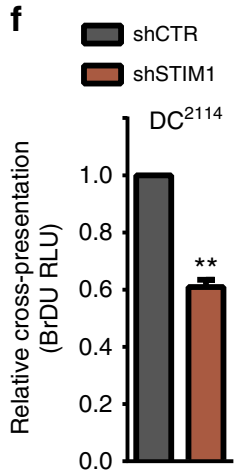

Fig. 1 STIM1 promotes cross-presentation in vivo and in vitro. a Representative flow cytometry gating strategy of cells isolated from draining (DL) and non-draining (NDL) lymph nodes of mice injected with 1.0 or $0.5 \%$ OVA-coated beads (OVAb), and with $1 \times 10^{6} \mathrm{CD} 45.1^{+}$OT-I cells. Out of $50,000 \mathrm{CD} 8 \alpha^{+}$ cells, the total number of CD45.1 $1^{+}$OT-I cells and their corresponding CFSE fluorescence were analysed. $\mathbf{b}$ OT-I cells were strongly decreased in DL but not $\mathrm{NDL}$ of CD45.2 $2^{+}$LysM-Cre; Stim $7^{f l / f l}$ mice as compared to Stim $7^{f l / f l}$ littermates (upper graph). Similarly, OT-I proliferation, as assessed by CFSE dilution, was significantly decreased in STIM1-deficient mice (lower graph). $N=3$ pairs of mice. c Cross-presentation of OVA antigens to OT-I cells by BMDCs exposed to OVAb ( $4 \mathrm{~h})$ was quantified as the BrDU incorporation after $72 \mathrm{~h}$ of co-culture. Stim 1 ablation reduced cross-presentation in vitro, both in immature BMDCs and in cells matured with $1 \mu \mathrm{gL}^{-1} \mathrm{LPS}$ or $0.1 \mu \mathrm{M} \mathrm{CPG}$, at varying OT-I:BMDC (T:DC) ratios. $N=3$, in triplicate wells. Ratio 1:1 T:DC in unstimulated WT cells $=1$. d Pre-incubation with $40 \mu \mathrm{M}$ BAPTA-AM or $1 \mu \mathrm{M}$ Xesto reduced cross-presentation, similar to known inhibitors $0.2 \mathrm{nM}$ ConcA or $10 \mu \mathrm{M} \mathrm{DPI}$, applied as positive controls. $N=3$, in triplicate wells. Control DMSO condition =1. e Incubation with varying doses of the OVA(257-264) fragment SIINFEKL (1 h) induced similar levels of cross-presentation in WT and STIM1-deficient BMDCs. $N=3$, in triplicate wells. $\mathbf{f}$ Reduced crosspresentation was also observed in DC 2114 + shSTIM1, as compared to control (+shCTR) as assessed with BrDU. Phagocytic targets added at 20:1 targets: cells. $N=3$, in triplicate wells. Error bars are means \pm SEM, $p^{\star}<0.5,{ }^{\star \star} p<0.1,{ }^{\star \star \star} p<0.01$ using a two-way ANOVA and Sidak's post test for $\mathbf{b}, \mathbf{c}$ and $\mathbf{e}$, and a Student's $t$-test for $\mathbf{d}$ and $\mathbf{f}$ 
a
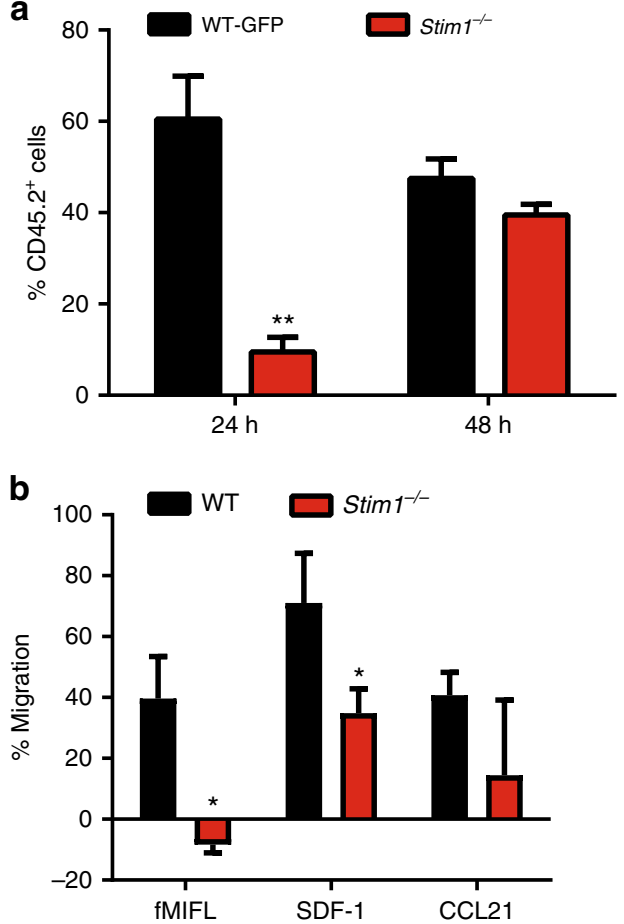

C

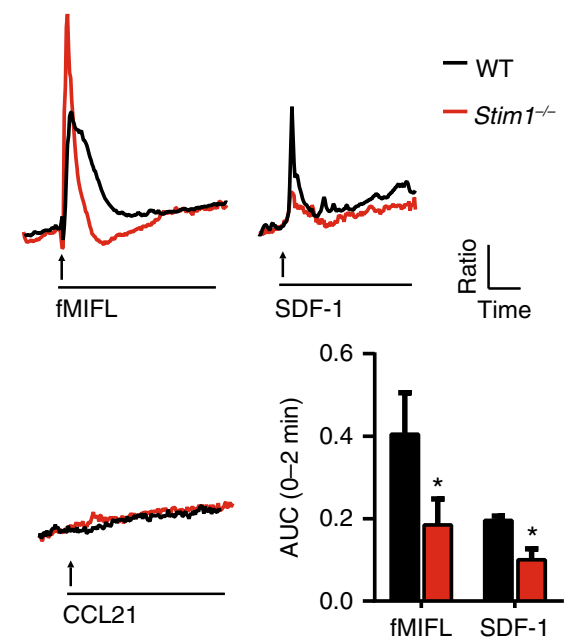

Fig. 2 STIM1 promotes $\mathrm{Ca}^{2+}$-dependent migration in vivo and in vitro. a CD45.1 congenic wild-type hosts were injected with a mixture of $1 \times 10^{6}$ wild-type GFP+ (WT-GFP), $1 \times 10^{6} \mathrm{Stim}^{-/-}$BMDCs and 0.5\% OVAb. DL were isolated and the relative percentage of WT:Stim1 $1^{-/-}\left(\mathrm{CD} 11 \mathrm{c}^{+}, \mathrm{CD} 45.2\right.$ ${ }^{+}$) cells analysed by flow cytometry. Full gating strategy is shown in Supplementary Fig. 2a. The proportion of Stim $1^{-1-}$ cells was significantly reduced $24 \mathrm{~h}$ but not $48 \mathrm{~h}$ after injection. $N=4$ pairs of mice. $\mathbf{b} A 24 \mathrm{~h}$ transwell migration assay revealed $40-65 \%$ increases in migration towards fMIFL, SDF-1 and CCL21 chemoattractants in WT cells. Stim1 ${ }^{-1-}$ BMDC showed reduced migration towards $\mathrm{FMIFL}$ and SDF-1 but not CCL21 (all applied at $10^{-6} \mathrm{M}$ ) as compared to WT. Full gating strategy is shown in Supplementary Fig. 2b. $\mathrm{N}=3$ in triplicate wells. $\mathrm{C} \mathrm{Ca}^{2+}$ transients induced by acute exposure (arrows) of chemoattractants, as measured using Fura-2 (traces representative average of 14-22 cells). Quantification of the area under the curve (AUC) of the first 2 min after chemoattractant addition (right bottom panel) revealed significantly lower $\mathrm{Ca}^{2+}$ entry in Stim1 ${ }^{-/-}$ BMDCs in response to $\mathrm{fMIFL}$ and SDF-1, while no $\mathrm{Ca}^{2+}$ entry in response to CCL21 was detected for either genotype. $N=4$ (coverslips each) containing: 68/66/72 (WT) or 47/56/52 (Stim1 ${ }^{-/-}$) cells for fMIFL/SDF$1 / C C L 21$. Error bars are means + SEM, ${ }^{\star} p<0.5,{ }^{\star *} p<0.1$ using a two-way ANOVA and Sidak's post test for $\mathbf{a}$ and a Student's $t$-test for $\mathbf{b}$ and $\mathbf{c}$

OVAb. DLs were collected at 24 and $48 \mathrm{~h}$ post injection, and the $\mathrm{CD} 45.1^{-} \mathrm{CD} 45.2^{+}$population analysed for GFP expression. The percentage of GFP-expressing WT cells in DLs was strongly and significantly higher at $24 \mathrm{~h}$ post injection, whereas the initial 1:1 ratio was nearly recovered at $48 \mathrm{~h}$ (Fig. 2a, Supplementary Fig 2a). BMDC migration was next examined in vitro using transwell chambers and the chemoattractant fMIFL to mimic microbial peptides found at the site of infection, SDF-1 (also known as CXCL12), a chemokine expressed by lymphatic vessels ${ }^{19}$, or CCL21 (also known as Exodus-2 or SLC), the major chemokine eliciting DC homing to lymph nodes (Fig. 2b, Supplementary Fig. 2b). No differences between baseline levels of migration between WT and Stim1 $1^{-/}$cells were observed, and surface expression of the SDF-1 and CCL21 receptors CXCR4 and CCR7 was similar in WT and Stim $1^{-/}$BMDCs matured or not with CpG, LPS, or OVAb (Supplementary Fig. 2c, d). WT cells showed strong, moderate and no $\mathrm{Ca}^{2+}$ transients evoked in response to fMIFL, SDF-1, and CCL21, respectively. STIM1-deficient cells displayed a brief $\mathrm{Ca}^{2+}$ elevation characteristic of $\mathrm{ER}^{-\mathrm{Ca}^{2+}}$ release by InsP $\mathrm{P}_{3}$-generating receptors, with an impaired delayed elevation characteristic of SOCE, resulting in a $\sim 50 \%$ reduction of the integrated $\mathrm{Ca}^{2+}$ response (Fig. 2c). Interestingly, the size of the migration defect was congruent with the size of the induced $\mathrm{Ca}^{2+}$ transients evoked by the same concentration of each chemoattractant $\left(10^{-6} \mathrm{M}\right)$ in STIM1-deficient cells, with fMIFL evoking the largest transients and the greatest migration defect, while CCL21 recruited STIM-deficient cells effectively without evoking $\mathrm{Ca}^{2+}$ responses (Fig. 2b, c). Preserved CCL21 signalling explains the delayed migration of STIM1-deficient DCs to lymph nodes and suggests that the CCL21-evoked $\mathrm{Ca}^{2+}$ transients reported in $\mathrm{DCs}^{20}$ might depend on other factors such as prostaglandins ${ }^{21}$. Together, these results imply that impaired $\mathrm{Ca}^{2+}$-dependent DC migration contributes to defective functional cross-presentation in vivo by delaying the recruitment of DCs to the site of injection and their subsequent rapid migration to lymph nodes. However, other factors are likely to contribute, since DCs eventually accumulate in lymph nodes.

Stim1 ablation impairs global and localized $\mathrm{Ca}^{2+}$ signals. SOCE can be functionally measured in isolated cells as the rate of $\mathrm{Ca}^{2+}$ entry after internal $\mathrm{Ca}^{2+}$ stores are depleted in the absence of external $\mathrm{Ca}^{2+}$. To further characterize the $\mathrm{Ca}^{2+}$ signalling defects of STIM1-deficient DCs, we used the SERCA inhibitor thapsigargin $(\mathrm{Tg})$ to passively deplete $\mathrm{ER} \mathrm{Ca}^{2+}$ stores and assess global SOCE. Stim 1 ablation reduced SOCE by $\sim 70 \%$ in both immature and mature BMDCs (Fig. 3a), a defect that could not be accounted for by changes in ORAI1 or STIM2 expression (Supplementary Fig. 3a). STIM1 knockdown also reduced SOCE by $70 \%$ in $\mathrm{DC}^{214}$ cells (Supplementary Fig. $3 \mathrm{~b}$ ), and $\mathrm{Ca}^{2+}$ responses evoked by platelet activating factor (PAF) in BMDCs were markedly impaired regardless of maturation (Fig. 3b, Supplementary Fig. 3c). In addition to global $\mathrm{Ca}^{2+}$ signals, localized $\mathrm{Ca}^{2+}$ hotspots occur during phagocytosis in macrophages, neutrophils and phagocytic fibroblasts ${ }^{17}, 22$. In fibroblasts, these signals coincide in time and space with the STIM1-mediated tethering of ER cisternae to phagosomes, reflecting both ER $\mathrm{Ca}^{2+}$ release as well as the opening of phagosomal $\mathrm{Ca}^{2+}$ channels ${ }^{17}$. We therefore checked whether periphagosomal $\mathrm{Ca}^{2+}$ hotspots and ER-phagosome MCS (ER-Ph MCS) were also present in DCs. Indeed, periphagosomal $\mathrm{Ca}^{2+}$ hotspots were observed in BMDCs exposed to OVAb for $30 \mathrm{~min}$ and were reduced by $\sim 50 \%$ upon Stim1 ablation (Fig. 3c, d). Periphagosomal $\mathrm{Ca}^{2+}$ hotspots were observed even after $90 \mathrm{~min}$ of OVAb exposure, although their frequency was reduced and their occurrence was no longer STIM1 dependent (Fig. 3c). SOCE channel inhibition with 
a
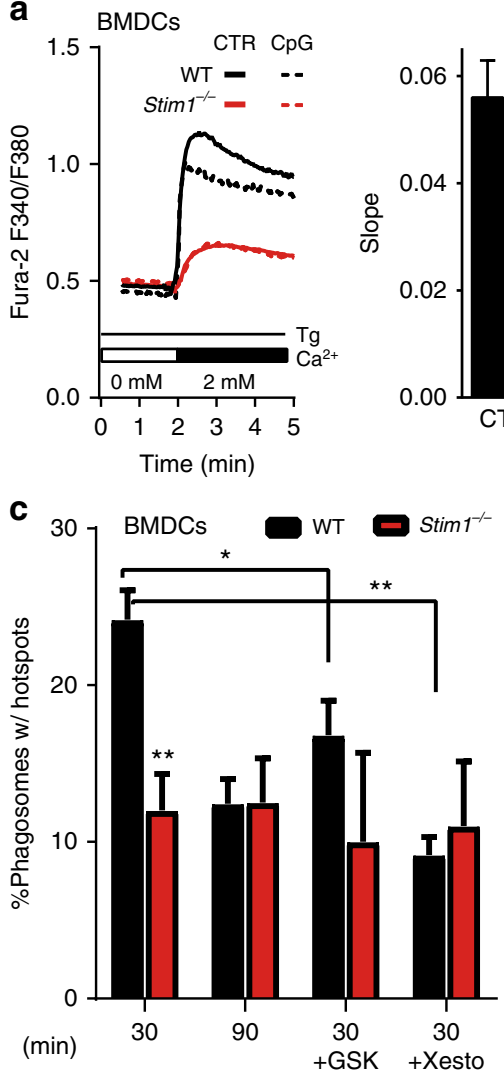

b

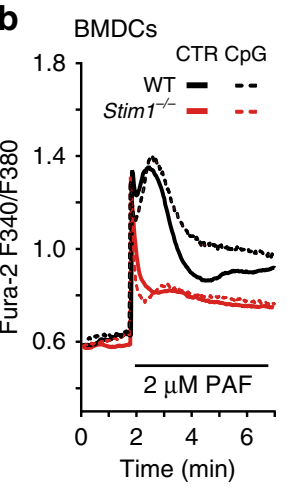

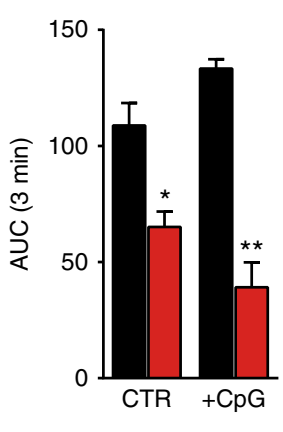
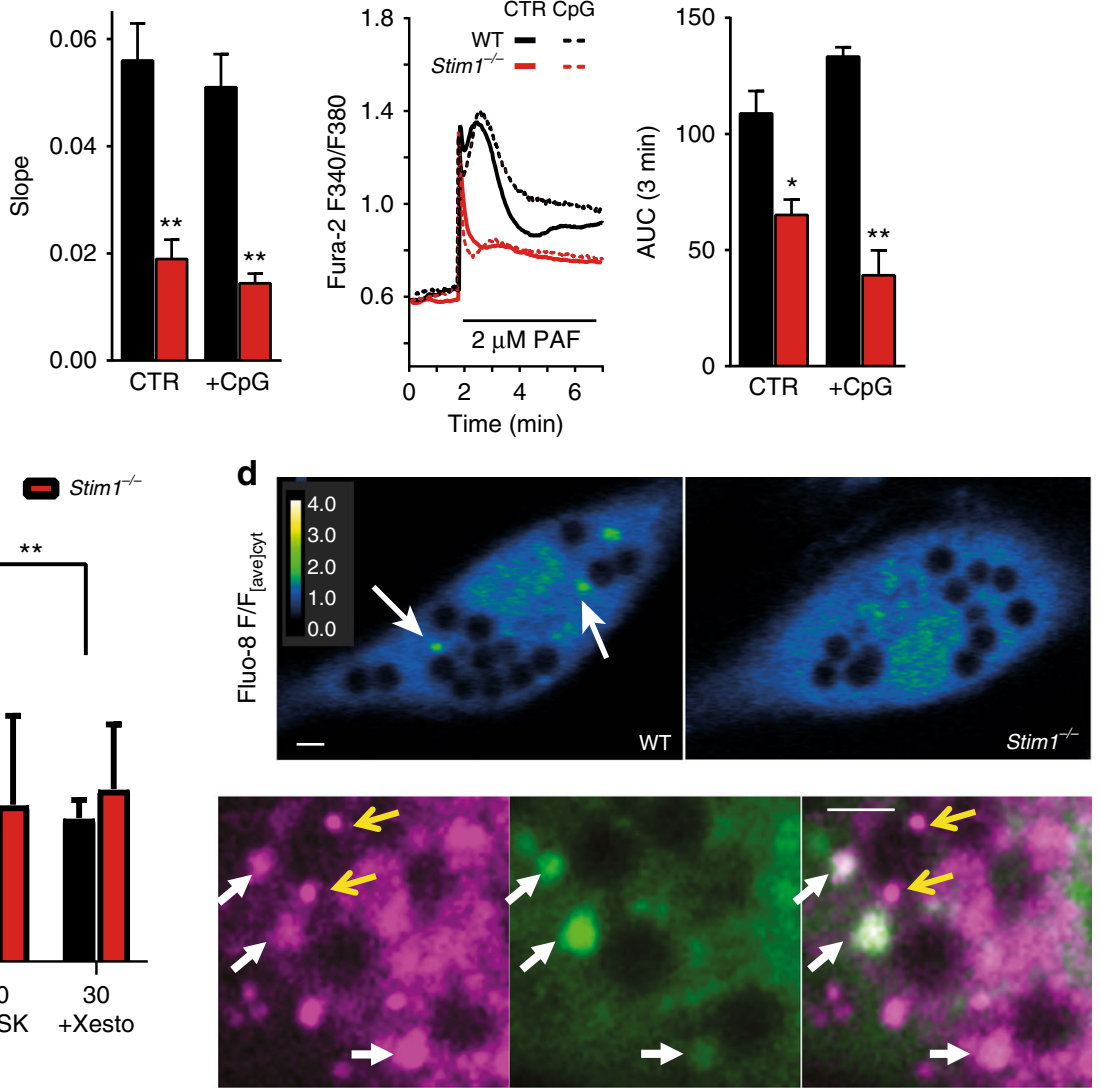

e

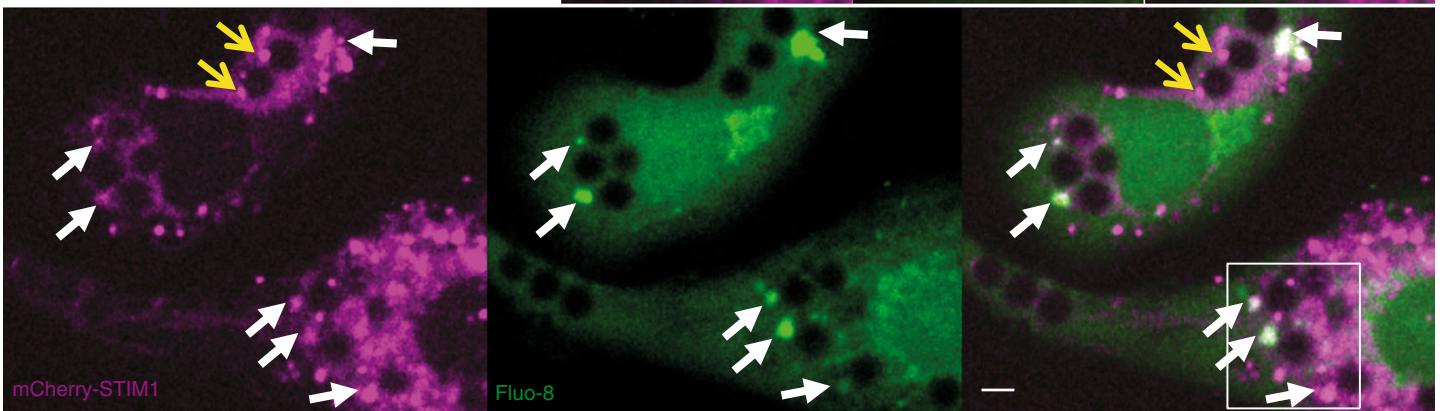

Fig. 3 STIM1 localizes near phagosomes and promotes global and local $\mathrm{Ca}^{2+}$ signals in BMDCs. a, b Stim1 ablation strongly decreased SOCE in immature and CpG-matured BMDCs, measured as the slope of $\mathrm{Ca}^{2+}$-re-entry after store depletion with $1 \mu \mathrm{M} \mathrm{Tg}$ in $\mathrm{Ca}^{2+}$-free medium and 2 mM Ca ${ }^{2+}$ re-addition $\mathbf{a}$, or as the area under the curve (AUC) of the first $3 \mathrm{~min}$ after acute exposure to the agonist PAF $(2 \mu \mathrm{M}, \mathbf{b})$. Traces are averages of $11-23$ cells. $N=10 / 6 ; 3 / 4$ (coverslips each) containing 129/119; 26/70 (WT) or 139/98; 67/97 (Stim1-/-) cells for Tg: CTR/CpG; PAF: CTR/CpG. c, d Localized Ca ${ }^{2+}$ signalling near phagosomes (green dots, arrows, d) was measured in BMDCs loaded with $4 \mu \mathrm{M}$ Fluo- 8 and $2.5 \mu \mathrm{M}$ BAPTA after 30 min or 90 min of exposure to targets, and in the absence or presence of $10 \mu \mathrm{M} \mathrm{GSK}$ or $1 \mu \mathrm{M}$ Xesto after $30 \mathrm{~min}$ (c). Stim1 ablation reduced periphagosomal Ca ${ }^{2+}$ hotspots at 30 but not 90 min, and both GSK and Xesto reduced hotspot frequency in WT but not Stim $1^{-/-}$cells (c). N=5/4;7/4;5/3;6/4 coverslips representing 870/794; 1916/1154; 898/814; 1124/1372 phagosomes; 196/178; 370/238; 270/265; 375/211 cells for WT/Stim1-/- 30;90:30+GSK;30+Xesto. The colour-coded ratio images are Fluo-8 fluorescence/average cytosolic Fluo-8 fluorescence and show representative hotspots (d). e BMDCs transduced with mCherry-STIM1 (magenta) and loaded with Fluo-8 (green) as above display periphagosomal accumulations of STIM1 fluorescence that co-localize with $\mathrm{Ca}^{2+}$ hotspots (white arrows), as well as puncta that show no $\mathrm{Ca}^{2+}$ activity (yellow arrows). 20:1 target:cell ratio. Bars $=3 \mu \mathrm{m}$. Error bars are means $+\mathrm{SEM}$, ${ }^{\star} p<0.5,{ }^{\star \star} p<0.1$ using a Student's $t$-test

GSK7975A (GSK, $10 \mu \mathrm{M})$ added concomitantly with OVAb, and Ins $\mathrm{P}_{3} \mathrm{R}$ inhibition with Xesto $(1 \mu \mathrm{M}) 20$ min after OVAb addition, reduced hotspot frequencies in WT but not STIM1-deficient cells (Fig. 3c). Although Xesto may affect STIM1 recruitment to phagosomes by preventing ER store depletion, these data suggest that both $\mathrm{Ca}^{2+}$ release from phagosomes as well as $\mathrm{Ca}^{2+}$ release from recruited ER stores contribute to local $\mathrm{Ca}^{2+}$ signals. In addition, in transduced BMDCs, periphagosomal mCherrySTIM1 puncta co-localized with periphagosomal hotspots
(Fig. 3e, white arrows). Periphagosomal STIM1 puncta that were not associated with $\mathrm{Ca}^{2+}$ hotspots were also observed (Fig. 3e, yellow arrows), but the majority of hotspots co-localized with STIM1 clusters. Immunostainings confirmed that STIM1 puncta were observed near phagosomes (Supplementary Fig. 4a). Similar to neutrophils, ER cisternae closely apposed $(<30 \mathrm{~nm})$ to phagosomes were observed in BMDCs exposed to OVAb for $30 \mathrm{~min}$, as visualized using both classic transmission electron microscopy (Supplementary Fig. 4b) as well as focused-ion-beam scanning 
a

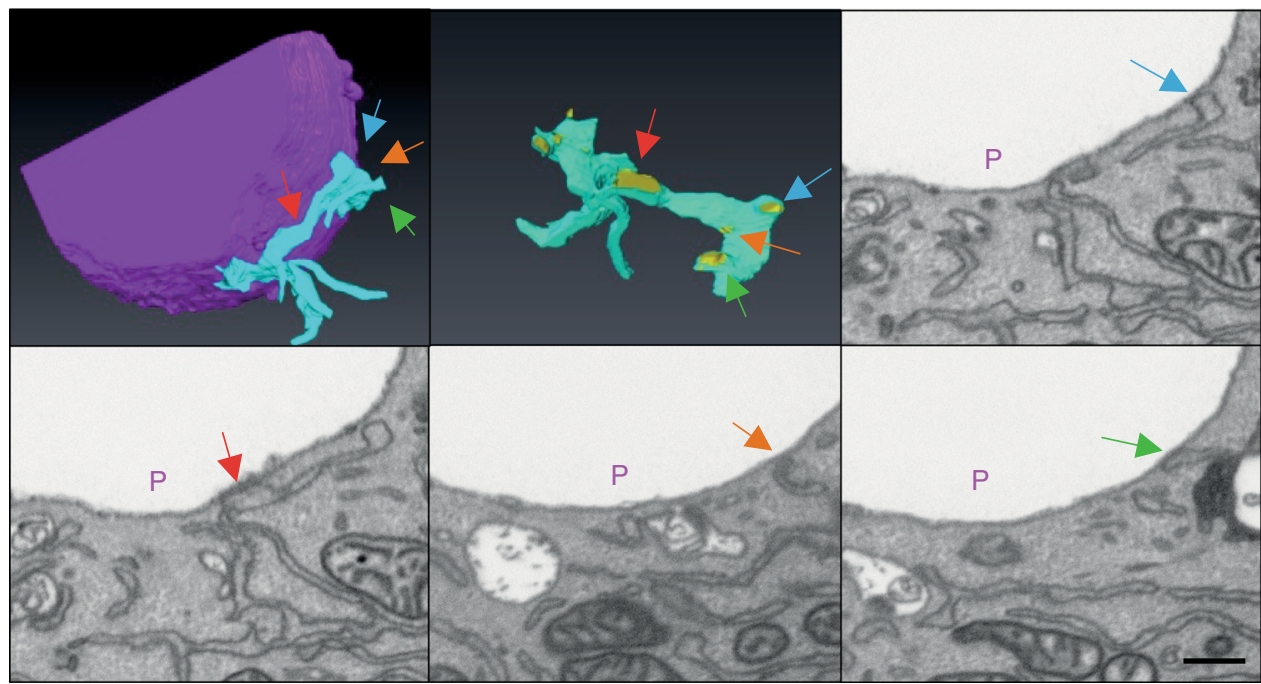

b

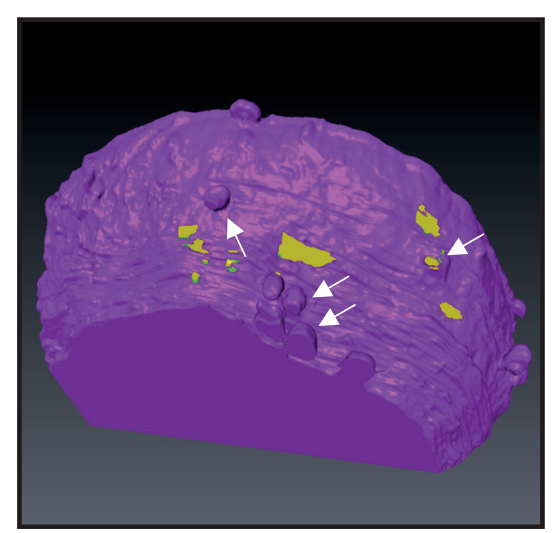

C

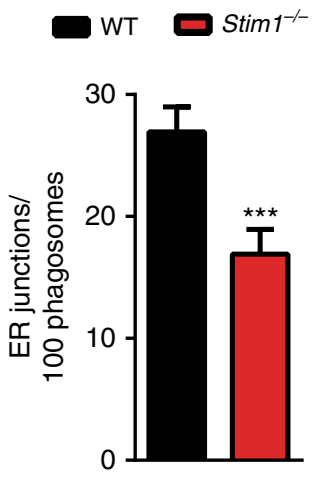

Fig. 4 STIM1 promotes ER-Phagosome membrane contact sites. a 3D reconstruction (top left and middle) of FIB-SEM images (top right and bottom) shows that contiguous ER membranes (light blue) make multiple contact sites (coloured arrows and yellow highlight) with a single phagosome (purple). b The vicinity of contact sites (yellow highlight) is associated with an increased occurrence of bulges in the phagosome surface away from the beads, reminiscent of vesicular fission or fusion activity (arrows). c Quantification of FIB-SEM slices revealed that Stim1 ablation reduced the frequency of ER-Ph MCS (also called ER junctions). $N=55 / 26$ slices representing a total of 170/35 phagosome cross-sections in WT/Stim1 ${ }^{-/-}$BMDCs respectively. OVAb added at 20:1 targets:cells, cells fixed $30 \mathrm{~min}$ after exposure to targets. Bar $=100 \mathrm{~nm}$. Error bars are means $+\mathrm{SEM},{ }^{\star} p<0.5$, ${ }^{\star \star} p<0.1$ using a Student's $t$-test

electron microscopy (FIB-SEM) (Fig. 4a-c). 3D reconstruction of FIB-SEM stacks confirmed that sites of ER-phagosome contact represented sections contiguous with the bulk ER (Fig. 4a). Interestingly, the $3 \mathrm{D}$ reconstruction also revealed that the same ER cisterna may display multiple sites of contact with the same phagosome (highlighted in yellow in Fig. 4a and Supplementary Movie 1). Additionally, whereas the phagosomal membrane closely follows the surface of the ingested beads for most of the phagosomal surface, in certain instances the region surrounding these ER-Ph MCS displayed multiple sites of bulging away from the bead that were reminiscent of vesicular trafficking (fusion or fission) activity (Fig. 4b, arrows). Quantification of EM slices revealed that similar to neutrophils ${ }^{17}$, Stim 1 ablation significantly reduced the number of ER-Ph MCS in BMDCs (Fig. 4c). Together, these data imply that STIM1 depletion has a major impact on global SOCE in DCs, as well as on localized $\mathrm{Ca}^{2+}$ signals mediated through ER-Ph MCS.

Stim1 is dispensable for DC maturation and phagocytosis. Previous reports suggest that DC differentiation and maturation are dependent on $\mathrm{Ca}^{2+}$ signalling ${ }^{22}$. BMDCs differentiated from bonemarrow precursors expressed high levels of CD11c, CD11b and low levels of F4/80, characteristic of conventional-like $\mathrm{DCs}^{23}$ (Fig. 5a,
Supplementary Fig. 5a). In both wild-type and Stim1 ${ }^{-/-}$BMDC cultures, $>90 \%$ of cells expressed CD11c and expression of all three markers were similar in both genotypes, as was surface levels of MHC-I, indicating that DC differentiation was unaffected by Stim 1 ablation (Fig. 5a). DC ${ }^{2114}$ cells were CD $8 \alpha^{+}, \mathrm{CD} 11 \mathrm{~b}^{-}, \mathrm{F} 4 / 80^{-}$and $\mathrm{B} 220^{-}$, (characteristic of $\mathrm{CD}^{+}$lymphoid-like $\mathrm{DCs}^{23}$ ), and expressed similar levels of CD11c when transduced with either shCTR or shSTIM1 (Supplementary Fig. 5b, c). Exposure of BMDCs to TLR ligands induces DC maturation characterized in part by an upregulation of cell-surface markers such as CD40, CD80, CD86 and MHC-II. When BMDCs were exposed to maturation stimuli CpG or LPS for $18 \mathrm{~h}$ the cell-surface expression of CD40, CD80, CD86 and MHC-II was similarly increased in both genotypes (Fig. 5b, Supplementary Fig. 2c). STIM1-silenced $\mathrm{DC}^{2114}$ cells were also equally capable of upregulating maturation markers in response to CpG (Supplementary Fig. 5d). We then checked whether acute administration of these TLR ligands evoked $\mathrm{Ca}^{2+}$ responses in immature cells. LPS evoked small $\mathrm{Ca}^{2+}$ transients in $\sim 30 \%$ of BMDCs and these signals were of lower amplitude in Stim $1^{-/}$cells, while $\mathrm{CpG}$ did not induce any detectable $\mathrm{Ca}^{2+}$ transient in either genotype (Supplementary Fig. 5e).

In neutrophils phagocytic ingestion is $\mathrm{Ca}^{2+}$ - and STIM1dependent ${ }^{17,24,25}$, and phagocytosis is $\mathrm{Ca}^{2+}$-dependent in $\mathrm{DCs}^{9}$, 
a
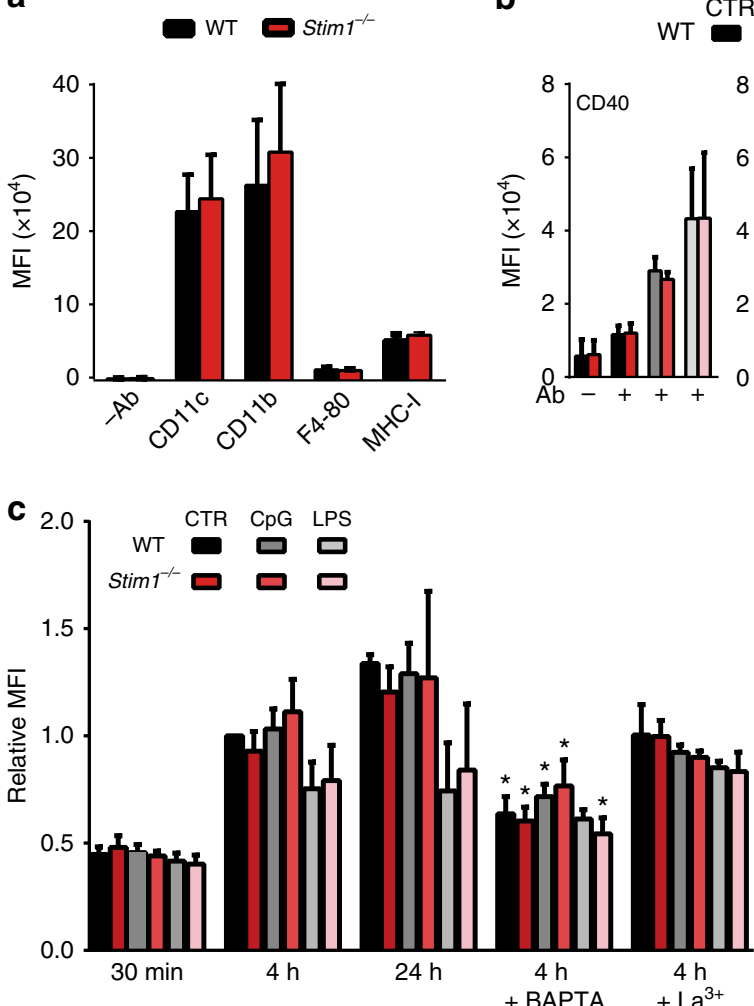

b
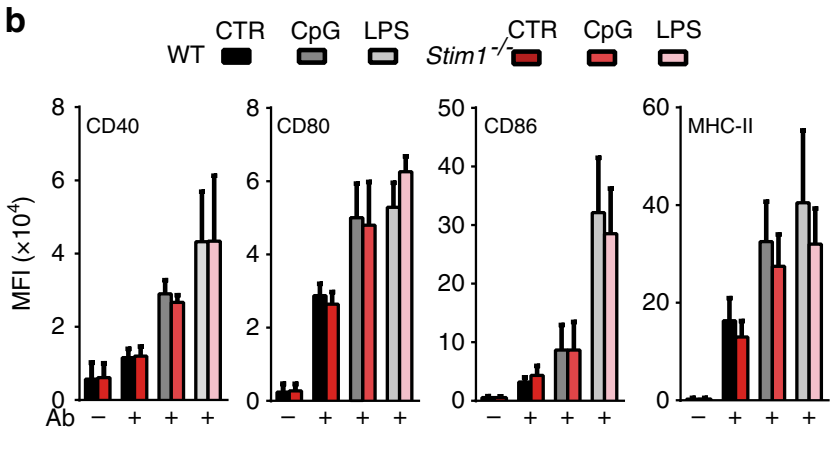

d

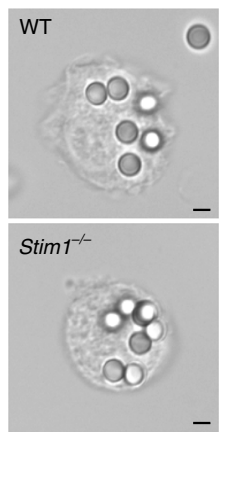

WT

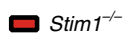

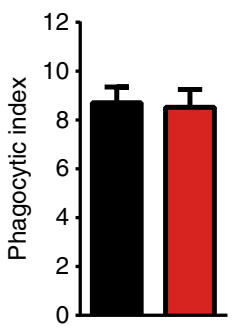

Fig. 5 Stim1 ablation does not affect BMDC differentiation, maturation, or phagocytic rate. a BMDCs express CD11c, CD11b, low levels of F4/80 and MHC-I to similar extents regardless of STIM1 expression. $N=8 / 5 / 5$ for CD11c/CD11b/F4/80. Values of cellular autofluorescence (-Ab) are shown for comparison. The gating strategy is shown in Supplementary Fig. 5a. b Expression of CD40, CD80, CD86 and MHC-II in immature cells or in cells matured with either CpG or LPS was similar in cells expressing or not STIM1. The full gating strategy is shown in Supplementary Fig. $2 \mathrm{c}$. N = 7/5/3 for CTR/CpG/ LPS for CD40/CD80, 8/5/7 for CD86 and 8/5/5 for MHC-II. c Phagocytosis of YG- Fluoresbrite-OVA-coated beads (OVAb) was decreased by loading cells with $40 \mu \mathrm{M}$ BAPTA but not by exposing cells to the non-specific SOCE blocker $\mathrm{LaCl}_{3}(50 \mu \mathrm{M})$, and was similar in immature and mature STIM1deficient cells as compared to cells from wild-type littermates. $N=4 / 5 / 3 / 3 / 3$ for $30 \mathrm{~min} / 4 \mathrm{~h} / 24 \mathrm{~h} / \mathrm{BAPTA} \mathrm{La}^{3+}$. The full gating strategy is shown in Supplementary Fig. 2c. d The absence of phagocytic defect in STIM1-deficient cells was confirmed by quantifying phagocytosis by microscopy. Phagocytic targets added at 20:1 targets:cells. $N=3 . \mathrm{MFI}=$ mean fluorescence intensity. Black bar $=3 \mu \mathrm{m}$. Error bars are means $+\mathrm{SEM}$, ${ }^{*} p<0.5$ using a Student's $t$ test

15. Phagocytic ingestion was therefore quantified by exposing BMDCs to OVA-coated Fluoresbrite-YG beads for $30 \mathrm{~min}, 4 \mathrm{~h}$ and $24 \mathrm{~h}$. While $\mathrm{Ca}^{2+}$ chelation by pre-incubation of cells with BAPTA-AM reduced phagocytosis by $\sim 40 \%$ in control WT cells, inhibition was less pronounced or not significant in mature cells, and neither Stim1 ablation nor the addition of the non-specific SOCE inhibitor $\mathrm{La}^{3+}$ significantly affected phagocytosis in either immature or mature BMDCs (Fig. 5c, Supplementary Fig. 2c). Furthermore, phagocytosis was unaltered in STIM1-deficient cells as assessed by microscopy (Fig. $5 \mathrm{~d}$ ). Together these data suggest that defects in DC differentiation, maturation or rates of phagocytic ingestion are unlikely to contribute significantly to the impaired cross-presentation of STIM1-deficient DCs.

Stim1 ablation does not alter phagosomal ROS or $\mathrm{pH}$. Several phagosomal maturation steps such as actin shedding, ROS production and fusion with granules or lysosomes are $\mathrm{Ca}^{2+}$-dependent in neutrophils and macrophages, 22 . In DCs, lower phagosomal proteolysis, higher phagosomal ROS and reduced phagosomal acidity correlate with higher cross-presentation ${ }^{26}$. In this context, reduced phagosomal ROS production, or increased acidity or proteolytic activity might lead to impaired crosspresentation in STIM1-deficient DCs. We therefore measured intracellular ROS production with dihydroethidium (DHE) in cells exposed to OVAb or zymosan particles (phagocytic targets known to elicit high levels of ROS), with the NADPH oxidase activator phorbol myristate acid (PMA) and inhibitor DPI serving as positive and negative controls. Intracellular ROS levels increased by 10-12-fold upon PMA or zymosan stimulation and by 4-6-fold upon exposure to OVAb, showing the highest level in cells matured with CpG (Fig. 6a). Extracellular ROS levels, measured with Amplex Rex, increased by 4-6-fold upon PMA exposure, whereas OVAb did not produce detectable extracellular ROS (Supplementary Fig. 6a). Stim1 ablation had no significant effect on ROS production in any condition tested (Fig. 6a, Supplementary Fig. 6a). BMDCs were also exposed to OVAb coupled to OxyBurst and Alexa-568, and imaged at $30 \mathrm{~min}$ and $90 \mathrm{~min}$ after bead addition. The ratio of OxyBurst to Alexa-568 fluorescence increased progressively following particle ingestion and was inhibited by DPI, yet again, no significant differences were detected (Fig. 6b).

We next examined whether phagosomal $\mathrm{pH}$ might be affected by Stim 1 ablation. Phagosomal $\mathrm{pH}$ was measured by exposing BMDCs to either FITC-coupled or to pHrodo-Red- and Alexa488-coupled zymosan. Zymosan was used instead of OVAb as FITC and pHrodo coupling to amine-functionalized beads was unstable. BMDCs were imaged after 30 and 90 min of exposure to zymosan, and pre-treatment with ConcA used to document V-ATPase-mediated acidification. Phagosomal $\mathrm{pH}$ was 

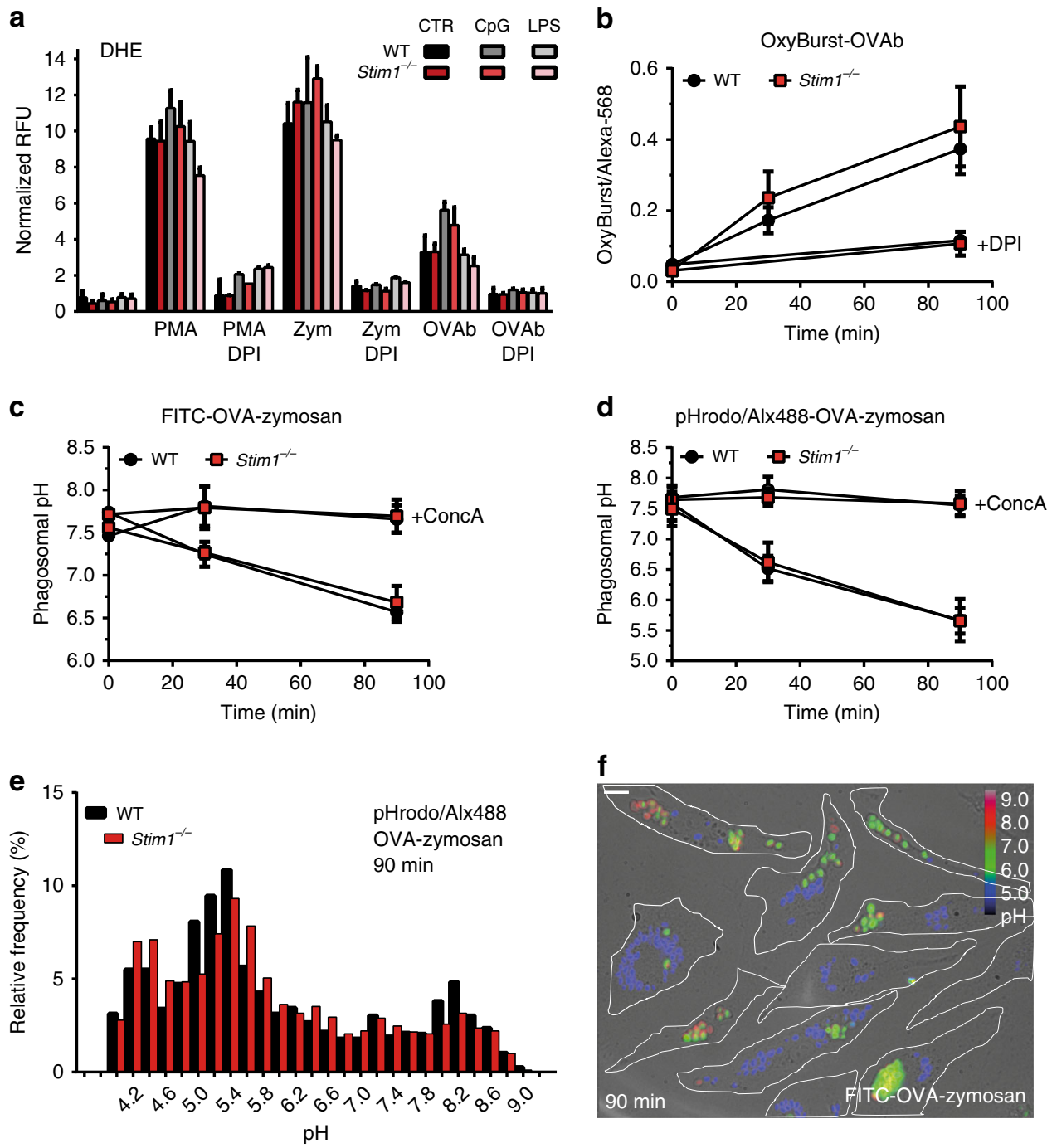

Fig. 6 Stim1 ablation does not affect ROS production or phagosomal pH. a Intracellular ROS production was measured in immature and CpG or LPSmatured BMDCs loaded with $30 \mu \mathrm{M} \mathrm{DHE}$, and exposed to OVA-coated beads (OVAb), zymosan (Zym) or $100 \mathrm{nM}$ PMA. N=4, triplicate wells. b ROS production during phagocytosis ( 30 and $90 \mathrm{~min}$ ) was assessed by exposing BMDCs to OVAb coupled to OxyBurst and Alexa-568. In a and b, DPI (10 $\mu$ M) blocked the DHE or OxyBurst signal, but no differences were detected upon Stim1 ablation. N=6/4/4 coverslips containing 84/54/58 (WT) or 85/50/53 $\left(S \mathrm{Stim}^{-/-}{ }^{-}\right.$) cells, for $30 \mathrm{~min} / 90 \mathrm{~min} / \mathrm{DPI}$. c, d Phagosomal acidification (30 and $90 \mathrm{~min}$ ) was measured using ratiometric or pseudo-ratiometric imaging by exposing cells to FITC-coupled (c) and pHrodo/Alexa-488-coupled (d) OVA-coated zymosan, respectively. Phagosomal acidification was blocked by ConcA $(0.2 \mathrm{nM})$, but there were no differences upon Stim1 ablation. $N=3$ coverslips for all conditions, comprising 3218/2826/1713/3533 (WT) or 2722/ 5295/2608/4438 (Stim1 $1^{-/-}$) FITC-phagosomes, and 2612/2194/700/746 (WT) or 1740/2218/670/862 (Stim1-/-) pHrodo phagosomes, for 30 min/ $90 \mathrm{~min} / 30 \mathrm{~min}$ ConcA/90 min ConcA. e Histogram of all combined 90 min pHrodo phagosomal pH values for WT and Stim1-/- BMDCs shows the broad and bimodal distribution of phagosomal $\mathrm{pH}$ in these cells. $\mathbf{f}$ Measurements of phagosomal $\mathrm{pH}$ by microscopy revealed the heterogeneity of the $\mathrm{pH}$ of individual phagosomes even within the same cell. Cells are outlined in white, the image is a merge of the brightfield and $480 / 440$ colour-coded ratio channels $90 \mathrm{~min}$ after addition of FITC-OVA-zymosan. $\mathrm{pH}$ values obtained from calibration curves (Supplementary Fig. $6 \mathrm{~b}$ ) are matched to the ratio colour-coded bar. Phagocytic targets added at 20:1 targets:cells. White bar $=10 \mu \mathrm{m}$. Error bars are means \pm SEM

determined from in situ calibration curves (Supplementary Fig. 6b) and measurements with FITC and pHrodo were made in parallel, using cells originating from the same mice. BMDCs showed a broad distribution of phagosomal $\mathrm{pH}$ ranging from very acidic to very basic ( $\sim \mathrm{pH} 4-9)$, displaying a roughly bimodal distribution, with an average $\mathrm{pH}$ that steadily decreased over the course of the recordings in a ConcA-sensitive manner (Fig. 6c-e). Phagosomes within the same cell had widely differing $\mathrm{pH}$ values, indicating that heterogeneity is determined at the level of single phagosomes (Fig. 6f). Regardless of the dye used, the phagosomes of Stim $1^{-/-}$BMDCs displayed an average $\mathrm{pH}$ similar to WT cells $(6.57 \pm 0.11$ vs. $6.68 \pm 0.19$ and $5.67 \pm 0.34$ vs. $5.66 \pm 0.21$ after $90 \mathrm{~min}$ for WT and Stim $1^{-/-}$cells using FITC and pHrodo respectively, Fig. $6 \mathrm{c}, \mathrm{d}$ ). Endosomal $\mathrm{pH}$ was additionally assessed by loading cells with Alexa-568 and FITC-coupled dextran. Although a trend for a more alkaline endosomal $\mathrm{pH}$ in Stim1 ${ }^{-/-}$ cells was apparent, the difference was not significant (Supplementary Fig. 6c).

Stim1 ablation impairs phagosomal proteolysis and fusion. We next measured phagosomal proteolysis, using beads coated with Alexa-568 and DQ-OVA, a protease probe consisting of OVA molecules heavily labelled with self-quenching BODIPY dye, which becomes brightly fluorescent upon OVA hydrolysis ${ }^{27}$. DQOVA fluorescence increased progressively, and green fluorescence 

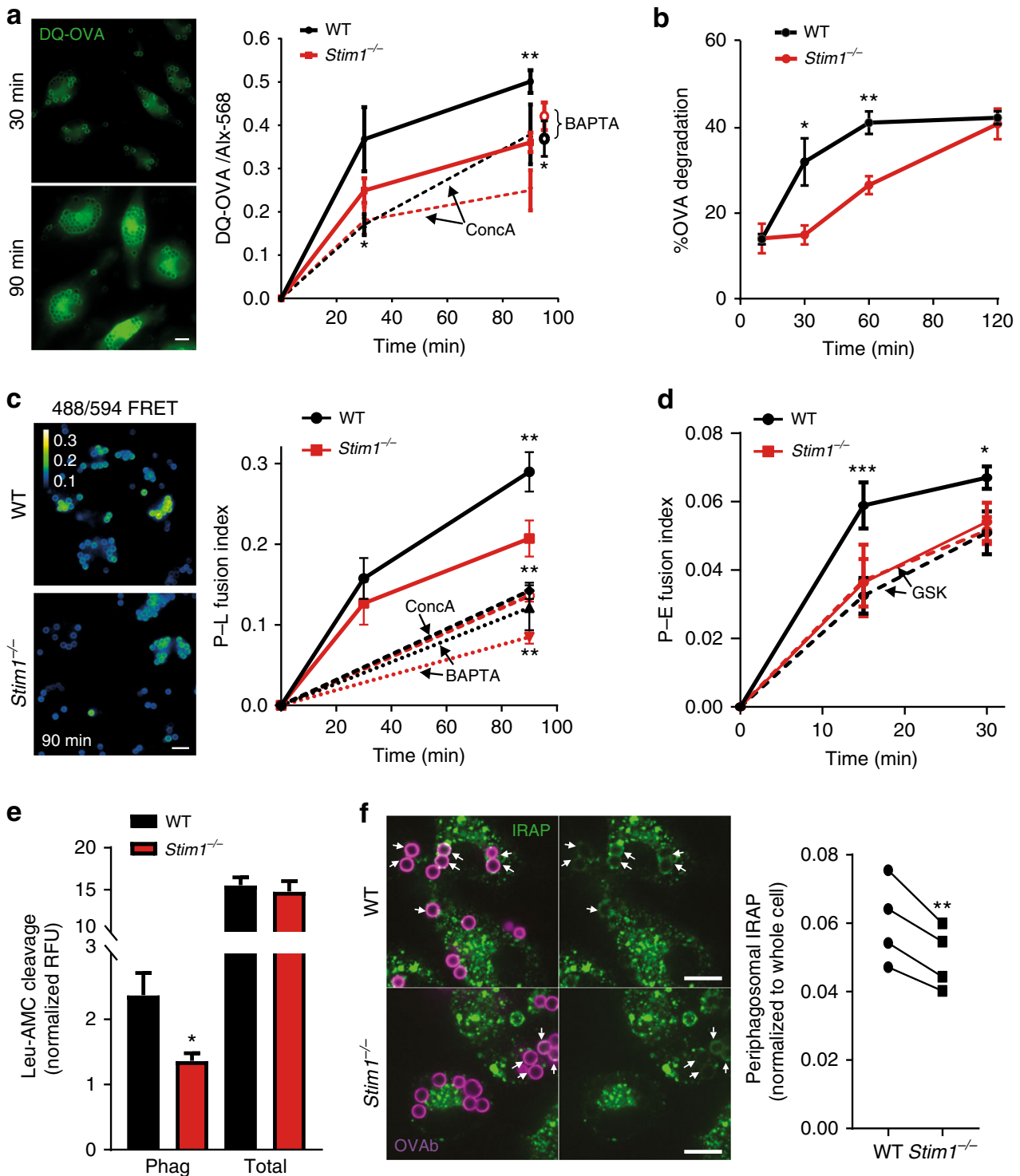

Fig. 7 STIM1 promotes phagosomal proteolysis and endomembrane fusion. a Proteolysis was measured in BMDCs exposed to DQ-OVA-Alexa-568 beads. STIM1-deficient cells showed lower levels of DQ-OVA fluorescence at 90 min (right panel). Pre-incubation with BAPTA-AM (40 $\mu$ M) reduced WT proteolysis to levels similar to STIM1-deficient cells. Lines representing the BAPTA condition are omitted and 90 min points are displaced to the right for clarity. ConcA ( $0.2 \mathrm{nM}$ ) only partially inhibited proteolysis. $N=5 / 10 / 3 / 5 / 3$ (coverslips) comprising a total of 236/1050/237/432/200 (WT) or 264/975/218/405/217 (Stim1 ${ }^{-/-}$) cells for 30/90/30 + ConcA/90 + ConcA/90 + BAPTA(min). b OVA degradation was measured with anti-OVA immunostainings of isolated phagosomes by flow cytometry. Values are \% OVA degradation. Full gating strategy is shown in Supplementary Fig. 7a. Proteolysis was decreased at 30 and 60 min after ingestion. $N=3$. c Phago-lysosome (P-L) fusion was measured by exposing Alexa-488-OVAb to cells loaded with lysosomal FRET acceptor Alexa-594-HA. Colour-coded images (left) show the FRET signal at 90 min for WT and STIM1-deficient cells. P-L fusion indices are matched to the colour-coded bar. P-L fusion was decreased in STIM1-deficient cells as compared to WT at 90 min, whereas BAPTA-AM loading further decreased P-L fusion and eliminated differences between WT and Stim ${ }^{-1}{ }^{-}$cells. ConcA decreased P-L fusion to similar levels as BAPTAloaded cells. $N=3 / 4 / 4 / 3$ (coverslips) comprising 470/1009/1691/797 (WT) or 643/1314/1617/503 (Stim1 $1^{-/-}$) phagosomes for 30/90/90 + ConcA/ 90 + BAPTA ( $\mathrm{min}$ ). d Phago-endosome (P-E) fusion was measured by exposing Alexa-488-OVAb to cells loaded with endosomal FRET acceptor Alexa594-dextran. P-E fusion was decreased in STIM1-deficient cells as compared to WT. Addition of GSK $(10 \mu M)$ eliminated differences in P-E fusion. $N=3$ (coverslips) comprising a total of 213/244/144/146 (WT) or 210/154/233/253 (Stim1 ${ }^{-/}$) ) cells for 15/30/15 + GSK/30 + GSK (min). Phagocytic targets added at 20:1. e Leu-AMC fluorescent substrate cleavage was reduced in phagosomes isolated from STIM1-deficient cells but not in whole-cell lysates, $N=$ 3. f Periphagosomal IRAP, quantified from single confocal slices, was reduced in STIM1-deficient cells. $N=4$ (coverslips). White bars $=10 \mu \mathrm{m}$. Error bars are means \pm SEM. ${ }^{\star} p<0.5,{ }^{\star \star} p<0.01$ using a two-way ANOVA and Sidak's post test for a-d and a Student's $t$-test for $\mathbf{e}$ and $\mathbf{f}$

leaked from the phagosome into the cytosol, indicating that proteolysis occurred (Fig. 7a). Contrary to macrophages ${ }^{27}$, phagosomal proteolysis reported by this assay was only partially inhibited by ConcA (Fig. 7a). Stim1 ablation significantly decreased DQ-OVA fluorescence by $\sim 30 \%$ at $90 \mathrm{~min}$. This effect tended to persist in the presence of ConcA, although the difference here was not significant $(p=0.09)$ (Fig. 7a).
Pre-loading cells with BAPTA-AM reduced proteolysis in both genotypes to the levels of untreated Stim $1^{-/}$cells, indicating that STIM1 mediates $\mathrm{Ca}^{2+}$-dependent proteolysis in BMDCs (Fig. 7a). As an alternative approach, the levels of phagosomeassociated OVA was determined by quantifying anti-OVA immunostaining of isolated BMDC phagosomes ${ }^{28}$. OVA degradation was inhibited in STIM1-deficient cells at 30 and $60 \mathrm{~min}$ 
but recovered after $2 \mathrm{~h}$, indicating a partial or delayed OVA degradation defect (Fig. 7b, Supplementary Fig. 7a).

Since phagosome-to-cytosol transfer of peptides is suggested to be favoured by the delivery of ER proteins to phagosomes ${ }^{16,29}$, we investigated whether endosome-to-cytosol transfer was influenced by Stim 1 ablation as a proxy measure of phagosomal antigen transfer ${ }^{16}$. BMDCs were loaded with the $\beta$-lactamase FRET reporter CCF4 and then exposed to $\beta$-lactamase. Upon transfer to the cytosol, $\beta$-lactamase cleaved CCF4, reducing the FRET emission in cells incubated at $37^{\circ} \mathrm{C}$ with identical kinetics in WT and STIM1-deficient cells (Supplementary Fig. 7b, c). This suggests that defective phagosome-to-cytosol transfer likely cannot account for the decreased DQ-OVA signal and that delivery of the molecular machinery underlying phagosome-to-cytosol transfer may not be dependent on STIM1.

We then tested phago-lysosome fusion. BMDCs were pulsed with the FRET acceptor Alexa-568-HA for $3 \mathrm{~h}$ followed by an overnight chase to accumulate the dye in lysosomes ${ }^{30}$. OVAb coupled to the FRET donor Alexa-488 were then added for 30 and $90 \mathrm{~min}$. The phagosome-associated FRET signal increased from 0 to $90 \mathrm{~min}$ in a ConcA and BAPTA-dependent manner, confirming that phago-lysosome fusion is $\mathrm{Ca}^{2+}$-dependent in BMDCs (Fig. 7c). Interestingly, Stim1 ablation reduced phago-lysosome fusion by $\sim 30 \%$ at $90 \mathrm{~min}$ (Fig. 7c). Since OVA degradation was most strongly affected at earlier time points, we additionally tested whether endosome fusion might be defective, by pre-loading cells for 15 min with Alexa-594-dextran before exposing them to Alexa-488-OVAb and measuring the cellular FRET signal. Indeed, phago-endosome fusion was reduced in Stim $1^{-9-}$ cells at 15 and $30 \mathrm{~min}$, with the effect most pronounced at $15 \mathrm{~min}$ (Fig. $7 \mathrm{~d}$ ). Addition of GSK $(10 \mu \mathrm{M})$ together with OVAb abolished differences between WT and Stim $1^{-/}$cells (Fig. 7d). These data suggest that reduced phago-lysosome and phago-endosome fusion contribute to reduced proteolysis in STIM1-deficient cells. Therefore, we tested whether the proteolytic activity of lysosomal or endosomal proteases might be affected. While there was a trend for decreased activity of the lysosomal hydrolases involved in antigen proces$\operatorname{sing}^{31}$ cathepsin B/L (CatB/L), cathepsin S (CatS) and asparagine endopeptidase (AEP), the differences were not significant (Supplementary Fig. 7d). On the other hand, leucyl aminopeptidase activity, characteristic of the endosomal protease insulinregulated aminopeptidase (IRAP) previously shown to be important for cross-presentation ${ }^{32}$, was reduced by $\sim 40 \%$ in phagosomes isolated from STIM1-deficient cells, but not in whole-cell lysates (Fig. 7e). Quantification of immunostainings revealed that periphagosomal IRAP was reduced by $\sim 20 \%$ (Fig. 7f). Together with the fact that $\mathrm{IRAP}^{+}$endosomes are partially co-labelled with fluorescent dextran internalized for 15 min (Supplementary Fig. 7e), these data indicate that reduced fusion of IRAP-containing endosomes contributes to the crosspresentation defect observed in STIM1-deficient cells, although a direct effect on the activity of phagosomal IRAP or of other leucyl aminopeptidases cannot be excluded.

\section{Discussion}

We report here that mice lacking STIM1 in the myeloid lineage fail to effectively cross-present phagocytosed antigens in vivo. The cross-presentation defect is recapitulated in vitro in DCs matured or not with TLR ligands, and is restricted to phagocytosed antigens, pointing to specific defects in phagocytic processing. Both global and localized $\mathrm{Ca}^{2+}$ signals are reduced in STIM1-deficient DCs. Surprisingly, but in line with a recent study ${ }^{15}$, DC differentiation, maturation and phagocytosis are not affected by Stim 1 ablation. Instead, defective cross-presentation is linked to two other impaired DC functions: defective in vivo migration, which correlates with reduced chemotaxis to $\mathrm{Ca}^{2+}$-mobilizing chemokines, and altered phagosomal maturation.

STIM1 deficiency reduces SOCE by $70 \%$ in both BMDCs and $\mathrm{DC}^{2114}$ cells indicating that STIM1 is the dominant isoform in DCs, and not STIM2 as proposed ${ }^{13}$. Since double Stim1;Stim2 ablation completely abolishes SOCE in DCs ${ }^{15}$ however, STIM2 still contributes to $\mathrm{Ca}^{2+}$ signalling in DCs. Similar to Vaeth et al, and unlike human DCs ${ }^{14}$, we observe that Stim1 is not required for the upregulation of DC maturation markers in mice, nor does it affect differentiation. Indeed, increased ROS production and upregulation of CCR7 and CXCR4, both events associated with DC maturation are additionally intact. This is in stark contrast to neutrophils derived from similar mouse genetic models, where phagocytosis ${ }^{17,25}$ and ROS production ${ }^{25}$ are both STIM1 dependent. These differences highlight the importance of cell-type dependence of $\mathrm{Ca}^{2+}$ signalling outcomes. Moreover, in contrast to RAW macrophages 33 and similar to B cells ${ }^{34}, \mathrm{CpG}$ does not induce detectable $\mathrm{Ca}^{2+}$ transients, while LPS induces small and variable $\mathrm{Ca}^{2+}$ transients in only $\sim 30 \%$ of cells. Thus, redundant $\mathrm{Ca}^{2+}$-dependent and independent pathways downstream of maturation stimuli appear sufficient to overcome a loss of STIM1 function in DCs.

We expected ROS production to be affected because NADPH oxidase activation is $\mathrm{Ca}^{2+}$ and STIM1 dependent in neutrophils ${ }^{35}$, and ROS production is required for effective cross-presentation (Fig. 1d and refs ${ }^{36-38}$ ). Surprisingly ROS production is unaffected in STIM1-deficient DCs suggesting that either sufficient $\mathrm{Ca}^{2+}$ signalling remains, or that oxidase activation is SOCEindependent in DCs. Phagosomal $\mathrm{pH}$ is intimately linked to phagosomal ROS production and depends on the delivery of $\mathrm{V}$ ATPase as well as HVCN1 proton channels $35,39,40$. In DCs phagosomal $\mathrm{pH}$ is controversial, with some groups reporting $\mathrm{pH}$ levels near 6.5 and others between 7 and 8 within the first $60 \mathrm{~min}$ of phagocytosis ${ }^{36,37}$. In all previous reports phagosomal $\mathrm{pH}$ was measured on cell populations. Here $\mathrm{pH}$ measurements are conducted on single phagosomes, using two independent probes, FITC and pHrodo, comparing cells originating from the same mice. No differences in subpopulations or average $\mathrm{pH}$ are detected between STIM1 and wild-type cells, but the two dyes yield an average $\mathrm{pH}$ differing by nearly 1.0 unit. This likely reflects the lower in situ pKa of pHrodo $(6.1 \pm 0.4$ vs. $7.6 \pm 0.3$ for FITC) and the increased sensitivity of pHrodo at low $\mathrm{pH}$ levels (Supplementary Fig. 6b), which indicates that pHrodo reports a more reliable $\mathrm{pH}$ estimate. On the other hand, the differences could also be related to the large variability in phagosomal $\mathrm{pH}$ that appears to be regulated at the level of single phagosomes. Shifts in the balance between the peaks of this bimodal, nonGaussian distribution between experimental conditions may explain why different groups report such disparate values for mean population measurements. Why DC phagosomes display such a large variability remains unclear but phagosomal ROS, which can be highly heterogenous ${ }^{41}$, and can inhibit V-ATPase recruitment ${ }^{35}$, is likely involved. Indeed, $\mathrm{Hvcn}^{-/-}$neutrophils, which suffer a $50 \%$ loss in ROS production, also display a bimodal distribution of phagosomal $\mathrm{pH}^{39}$, and thus it could be interesting to correlate ROS and $\mathrm{pH}$ measurements at the level of single phagosomes in future studies in DCs. It is interesting to note that ORAI channels are sensitive to extracellular acidity, being activated at alkaline $\mathrm{pH}$ while inhibited at $\mathrm{pH}<6^{42}$. The heterogeneity of phagosomal $\mathrm{pH}$ observed would thus allow phagosomes with robust ORAI activity to co-exist with phagosomes where ORAI is inhibited, and could explain why only a subset of STIM1-positive periphagosomal puncta coincide with $\mathrm{Ca}^{2+}$ hotspots. The identical $\mathrm{pH}$ profiles of STIM1 and wild-type 
cells however, indicate that altered $\mathrm{pH}$ cannot account for defects in cross-presentation.

Despite the lack of differences in either ROS or pH, STIM1deficient DCs display surprisingly lower levels of proteolysis, measured by two independent assays, as well as reduced leucyl aminopeptidase activity, characteristic of the endosomal protease IRAP previously implicated in cross-presentation ${ }^{32}$. Decreased proteolysis is associated with decreased phago-endolysosome fusion, decreased global and periphagosomal $\mathrm{Ca}^{2+}$ signals, and decreased frequency of ER-Ph MCS in STIM1-deficient cells. Although we cannot differentiate whether loss of global or local $\mathrm{Ca}^{+}$signals cause reduced fusion events, the high vesicular fusion/fission activity near ER-Ph MCS favours a model where local $\mathrm{Ca}^{2+}$ signals promote local fusion events. It should be noted that decreased cathepsin-dependent proteolysis and reduced phago-lysosome fusion correlated with higher, not lower, levels of cross-presentation in $\mathrm{DCs}^{38,43}$. Yet, some proteolysis must occur to generate peptides from whole OVA molecules and inhibiting proteolysis with the broad-spectrum inhibitor leupeptin reduces cross-presentation in some ${ }^{44-46}$ although not all ${ }^{47}$, 48 contexts. Indeed, in the companion study published in this same issue which analyses the function of UNC93B1, a novel STIM1 interactor ${ }^{49}$, Maschalidi et al. not only confirm a role for STIM1 in cross-presentation, but also observe that decreased proteolysis correlates with decreased cross-presentation. Proteolysis has therefore a bipartite effect on antigen processing, with both negative and positive effects on the MHC-I loading depending on its extent. Our data suggest that finely regulated levels of proteolysis must be reached to favour cross-presentation, and that this fine-tuning is achieved at least in part through $\mathrm{Ca}^{2}$ ${ }^{+}$-dependent regulation of endosome fusion and IRAP delivery.

We also considered that antigen export from phagosomes into the cytosol might be affected by STIM1 deletion. Phagosomal export was reported for some but not all antigen $s^{50}$ and cytosol transfer requires ER proteins such as Sec61, tapasin and TAP ${ }^{1}$. That STIM1 mediates close contacts between the ER and phagosomes argues that at least some ER proteins found on isolated phagosomes are derived from contacts, which may in turn facilitate the delivery of this ER-derived machinery. That the frequency of ER-Ph MCS was fivefold higher in WT DCs as compared to WT neutrophils, yet the frequency of periphagosomal $\mathrm{Ca}^{2+}$ signals is only half that of neutrophils ${ }^{17}$, as well as the observation of periphagosomal STIM1 puncta without associated $\mathrm{Ca}^{2+}$ signals (Fig. 3e), further support the idea that $\mathrm{ER}-\mathrm{Ph}$ MCS may have roles beyond $\mathrm{Ca}^{2+}$ signalling in DCs, and that they could partly mediate specialized DC functions that rely on ER proteins. However, no defects in cytosol transfer were observed. On one hand, ER contacts were only decreased by $\sim 35 \%$ upon Stim1 deletion, and potentially, sufficient activity remained to allow effective cytosol transfer. On the other hand, recent data suggests that different ER proteins such as tapasin ${ }^{16}$, MHC- $\mathrm{I}^{8}$ and Sec $61^{7}$ reach phagosomes through unconventional vesicular trafficking pathways involving the ERGIC, recycling endosome and possibly other compartments. These new findings on alternative trafficking raise the intriguing possibility that endomembrane trafficking is more generally non-canonical in DCs and that specialized compartments analogous to secondary and tertiary granules in neutrophils might exists in DCs. Indeed, a lysosome-like Rab34-dependent compartment delivers NADPH oxidase in $\mathrm{DCs}^{48}$. Since cross-presentation was more strongly impaired than phago-lysosome fusion, STIM1 might regulate alternative $\mathrm{Ca}^{2+}$-dependent trafficking pathways in a way that bypasses the need for cytosol transfer. The generation of better tools to manipulate these pathways will be required to gain a deeper understanding of alternative compartments and the signals that regulate their activity.
In summary, we show that STIM1 has an important and selective regulatory role on $\mathrm{Ca}^{2+}$-dependent processes that control phagosome maturation and migration in DCs, thereby impacting the efficiency of cross-presentation. STIM-gated ORAI channels are promising drug targets for treating autoimmune diseases $^{51}$, muscle defects ${ }^{52}$, skin disorders ${ }^{53,54}$ and cancer $^{55}$. In addition, activating ORAI1 through a genetically encoded lightinducible STIM1 fragment promotes tumour remission in a mouse model of DC cell-based immunotherapy ${ }^{56}$. Together with our data showing that either pharmacological or genetic manipulation of $\mathrm{Ca}^{2+}$ signalling can decrease cross-presentation, this suggests that delineating the precise role of SOCE in innate immune cells is highly relevant for the identification of potential risks and benefits associated with the development of SOCE blockers and activators designed for therapeutic use.

\section{Methods}

Reagents. The following antibodies (antibody Name/catalogue\#/dilution) were purchased from: Biolegend (USA)- PerCP/Cy5.5-anti-CD197 (also known as CCR7)/120115/1:100; PE-anti-CD184 (also known as CXCR4) L276F12/146505/ 1:100; APC-anti-F4/80 (BM8)/123115/1:400, APC-Rat-IgG2a, $\kappa$ Isotype Ctrl/ 400511/1:100; PE/Cy7-anti-CD11b (M1/70)/101225/1:200; PE-anti-CD86 (GL-1)/ 105007/1:200; PE/Cy7-anti-CD40 (3/23)/124621/1:100; APC-anti-CD80 (1610A1)/104713/1:100; Alexa-647-anti-I-Ab (also known as MHC-II, KH74)/115309/ 1:200; PE/Cy7-anti-CD8 $\alpha$ (53-6.7)/100721/1:200; APC-anti-CD45.1 (also known as PTPRC $^{\mathrm{a}}$, A20)/110713/1:100. BD Biosciences (USA): APC-anti-CD11c (HL3)/ 561119/1:100; anti-CD16/CD32 (2.4G2) "FcBlock"/553142/1:200; anti-STIM1/ 610954/1:100. Millipore (USA): anti-STIM1/AB9870/1:1000. eBiosciences (USA): PE-anti-CD11c (N418)/17-0114-81/1:100; APC-anti-CD45.2(104)/17-0454-81/ 1:100. Cell Signalling (USA): anti-STIM2/4917S/1:1000; anti-IRAP/6918/1:250. Sigma-Aldrich (Germany): anti-Orai1/O8264/1:500. Bio-Rad (USA): anti-mouseHRP/1706516/1:10,000; anti-rabbit-HRP/ 172101/1:10,000. Jackson ImmunoResearch (USA): Alexa-488-anti-mouse /715-545-150/1:800. Recombinant murine SDF-1 $\alpha$ (also known as CXCL12), recombinant murine CCL21 (also known as Exodus-2 or SLC), and recombinant murine GM-CSF were obtained from Peprotech (UK). Carboxyfluorescein succinimidyl ester (CFSE) was obtained from eBiosciences. Mouse shSTIM1 (TRC Clone ID: NM_009287.2-2712s1c1 Sequence: CCGGCCCTTCCTTTCTTTGCAATATCTCGAGATATTGCAAA-

GAAAGGAAGGGTTTTT TG) and shCTR (Non-target Control particles SCH002V) Mission shRNA Lentiviral Clones were purchased from Sigma-Aldrich. All lentiviral particles were produced in Lenti-X 293T cells using the Lenti-X HTS Packaging System (Takara, Japan) according to the manufacturer's instructions. Lentiviral titres were determined using the LentiX-p24 Rapid Titre ELISA kit (Takara). All cell culture reagents were obtained from ThermoFisher Scientific, and all chemicals were purchased from Sigma-Aldrich unless otherwise stated. mCherry-STIM1 ${ }^{57}$ cloned into pENTR1a was purchased from GenScript (USA). mCherry-STIM1 was then cloned into the $\mathrm{p} 2 \mathrm{~K} 7_{\mathrm{bsd}}$ lentiviral vector containing the ubiquitin promoter using the Gateway LR clonase (ThermoFisher Scientific, USA) following the protocol described in ref. ${ }^{58}$. OVA-beads were prepared by washing $3.0 \mu \mathrm{m}$ unlabelled or YG-Fluoresbrite polystyrene microspheres (Polysciences,

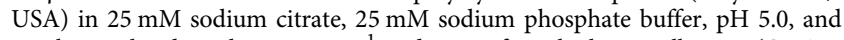
incubating beads with $20 \mathrm{mg} \mathrm{mL}^{-1}$ endotoxin-free chicken ovalbumin (OVA, InvivoGen, USA) overnight at $4^{\circ} \mathrm{C}$ on an end-over-end rotor, followed by three washes in sterile PBS (ThermoFisher).

Flow cytometry. Cells were washed once in ice cold FACS buffer (2\% BSA, $20 \mathrm{mM}$ EDTA in PBS), blocked with 1:200 FcBlock/FACS buffer $15 \mathrm{~min}$ on ice, incubated for 30 min-1 h with the indicated antibodies, and washed with FACS buffer. Fluorescence was analysed using an Accuri C6 flow cytometer and CFlow Plus software (BD Biosciences) unless otherwise indicated.

Mice, cells and transduction. Mice on a C57BL/6 background bearing a conditional knockout of the Stim 1 gene in the myeloid lineage were generated from LysM-Cre (B6.129P2-Lyz2 $2^{\text {tm1(cre)Ifo } / J^{59}}$, The Jackson Laboratory, USA) and Stim $\mathrm{f}^{\mathrm{fl}}$ ${ }^{l}{ }^{\prime}\left(\mathrm{B} 6(\mathrm{Cg})-\right.$ Stim $^{\text {tm1 Rao }} / \mathrm{J}^{60}$, (a kind gift from Dr. Masatsugu Oh-Hora) strains, and genotyped from ear biopsies using a KAPA Mouse Genotyping Kit (Sigma) according to the manufacturer's instructions, and the following primers and PCR conditions: For Stim1, CGATGGTCTCACGGTCTCTAGTTTC; AACGTCTTGCAGTTGCTGTAGGC; GGCTCTGCTGACCTGGAACTATAGTG and $94^{\circ} \mathrm{C} 3$ $\min , 94^{\circ} \mathrm{C} 7 \mathrm{~s}, 60^{\circ} \mathrm{C} 20 \mathrm{~s}, 72^{\circ} \mathrm{C} 25 \mathrm{~s}, 30$ cycles, $72^{\circ} \mathrm{C} 3 \mathrm{~min}$. For LysM-Cre: CCCAGAAATGCCAGATTACG, CTTGGGCTGCCAGAATTTCTC, TTACAGTCGGCCAGGCTGAC and $95^{\circ} \mathrm{C} 5 \mathrm{~min}, 95^{\circ} \mathrm{C} 45 \mathrm{~s}, 60^{\circ} \mathrm{C} 20 \mathrm{~s}, 72^{\circ} \mathrm{C} 25 \mathrm{~s}, 40$ cycles, $72^{\circ} \mathrm{C} 5 \mathrm{~min}$ in separate reactions, where $1 \%$ DMSO and $1.6 \%$ Perfect Match (Agilent) were added to the reaction of the mutant band. Bone marrow was isolated from sex and age-matched 6-16 week-old males and females. All animal manipulations were approved by the Geneva canton's Direction Générale de la Santé 
(authorizations GE/87/15 and GE/142/16) and performed in accordance to the guidelines of the animal research committee at the University of Geneva. BMDCs were generated by culturing bone marrow cells isolated from mouse femurs and tibias, where red blood cells had been removed by lysis in ammonium buffer (155 $\mathrm{mM} \mathrm{NH}_{4} \mathrm{Cl}, 10 \mathrm{mM} \mathrm{KHCO} 3,0.1 \mathrm{mM}$ EDTA), in DMEM (41965-039) supplemented with $10 \%$ endotoxin-free FCS, $50 \mu \mathrm{M} \beta$-mercaptoethanol, $1 \%$ sodium pyruvate, $1 \%$ penicillin/streptomycin and $20 \mathrm{ng} \mathrm{mL}^{-1}$ murine recombinant GMCSF. Cells were used between 8 and 15 days of culture. The purity of each culture was determined to be $>85 \% \mathrm{CD}_{11 \mathrm{c}^{+}}$by flow cytometry. The $\mathrm{DC}^{2114}$ cell line, derived from $\mathrm{C} 57 \mathrm{BL} / 6$ mice with an $\mathrm{H} 2-\mathrm{K}^{\mathrm{b}}$ haplotype ${ }^{18}$ was cultured in IMDM (31980) supplemented with $10 \%$ FCS, $50 \mu \mathrm{M} \beta$-mercaptoethanol, and $1 \%$ penicillin/streptomycin. Cells were tested for mycoplasma every six months. Maturation was performed by incubating cells in either $1 \mu \mathrm{g} \mathrm{mL}^{-1}$ LPS or $0.1 \mu \mathrm{M} \mathrm{CpG}$ (ODN 1826, InvivoGen) for $18 \mathrm{~h}$. Seeding cells on coverslips tended to activate them to variable extents, thus unless otherwise indicated cells seeded on coverslips were matured with $\mathrm{CpG}$. Lentivirus transduction was performed by centrifuging cells and viral particles at $5 \mathrm{MOI}$ in complete medium supplemented with $8 \mu \mathrm{g} \mathrm{mL}$ ${ }^{-1}$ polybrene at $500 \mathrm{~g}$ at $37^{\circ} \mathrm{C}$ for $1 \mathrm{~h}$. To produce $\mathrm{DC}^{2114}$ cells stably expressing shRNA, cells were cultured in $1 \mu \mathrm{g} \mathrm{mL} L^{-1}$ puromycin as of 2 days after transduction. Splenic OT-I cells were isolated from C57BL/6-Tg(TcraTcrb) $1100 \mathrm{Mjb} / \mathrm{J}$ mice $^{61}$ (Jackson) using the $\mathrm{CD} 8 \alpha^{+}$negative selection mouse $\mathrm{T}$ cell isolation kit (Miltenyi Biotec, Germany) according to the manufacturer's instructions. Congenic CD45.1 ${ }^{+}$ OT-I cells were obtained by breeding OT-I mice to the congenic CD45.1 strain B6. SJL-Ptprc ${ }^{\mathrm{a}}$ Pepc $/ \mathrm{BoyJ}^{62}$ (Jackson) and similarly isolating CD8 $\alpha^{+}$cells. Cell purity was verified to be $>95 \% \mathrm{CD} 8 \alpha^{+}, \mathrm{CD}^{-}$by flow cytometry.

In vivo cross-presentation. Three to four-month-old female $L y s M-C r e^{k i /+}$; Stim $1^{f l / f l}$ and otherwise wild-type Stim $1^{f / f l}$ littermates were anesthetized with isofluorane and injected with $50 \mu \mathrm{l}$ of $1.0 \%$ OVA-beads/PBS on the left and $0.5 \%$ OVA-beads/PBS on the right footpads, or with only PBS on both footpads as control. Twenty four hours later the mice were retro-orbitally injected with $1 \times 10^{6}$ CD45.1 $1^{+}$OT-I cells labelled with $3.5 \mu \mathrm{M}$ CFSE for $10 \mathrm{~min}$ and washed and resuspended in $50 \mu \mathrm{l}$ sterile PBS. After $72 \mathrm{~h}$, the mice were sacrificed and popliteal draining and axial/brachial non-draining lymph nodes were harvested in FACS buffer containing $2 \%$ FCS. Lymph nodes were mechanically crushed onto $40 \mu \mathrm{m}$ cell strainers and the cellular filtrate blocked and stained as described above with anti-CD8 $\alpha$-PE/Cy7 anti-CD45.1-APC antibodies. The total number of CD45.1 ${ }^{+}$ cells as well as their CFSE fluorescence was determined for 50,000 CD8 $\alpha^{+}$cells. \% Proliferation is defined at the percentage of cells showing diluted levels of CFSE fluorescence as compared to uninjected controls.

In vitro cross-presentation. BMDCs were seeded at 20,000 cells per well and $\mathrm{DC}^{2114}$ at 10,000 cells per well in black/clear-bottom 96-well plates (Greiner, for BrDU-based assays) or in round-bottom 96-well plates (Corning, for CFSE assays), and allowed to adhere for $24 \mathrm{~h}$. For 3:1 and 10:1 T cell:DC ratios, BMDCs were diluted accordingly. Cells were exposed to either OVAb at 20:1 ratio for $4 \mathrm{~h}$ or SIINFEKL peptide (InvivoGen) for $1 \mathrm{~h}$ at the given concentrations, before wells were washed in complete medium, and irradiated with 3000 cGy using a Gammacell 3000 Elan $\mathrm{Ce}^{127}$ irradiator (Best Theratronics, Canada). 20,000 (for BMDC) or 10,000 (for $\mathrm{DC}^{2114}$ ) OT-I cells were added to each well, and plates were incubated at $37^{\circ} \mathrm{C} / 5 \% \mathrm{CO}_{2}$ for $72 \mathrm{~h}$. T cell proliferation was assessed by BrDU incorporation using a Cell Proliferation ELISA, BrDU (Chemiluminescent) kit (Roche, Switzerland) according to the manufacturer's instructions and using a SpectraMAX Paradigm (Molecular Devices, USA) plate reader. Proliferation of OT-I cells labelled with CFSE was assessed as described above.

In vivo migration. BMDCs isolated from $L y s M$-Stim $1^{f / f l}$ and from Ubiquitin-eGFP mice (C57BL/6-Tg(UBC-GFP) 30 Scha/ $/{ }^{63}$, Jackson) were washed and resuspended in PBS containing $0.5 \%$ OVAb at $20 \times 10^{6}$ cells per mL each. In total, $50 \mu \mathrm{l}$ of cell/ bead mixture were injected into left and right footpads of congenic B6 CD45.1 ${ }^{+}$ mice. Popliteal lymph nodes were harvested $24 \mathrm{~h}$ or $48 \mathrm{~h}$ post injection and DCs were recovered from $\mathrm{LN}$ after digestion in an enzymatic mix containing collagenase $\mathrm{D}\left(1 \mathrm{mg} \mathrm{mL}^{-1}\right)$ and DNAse I $\left(10 \mu \mathrm{g} \mathrm{mL}^{-1}\right)$ (Roche) in HBSS. Total LN cells were incubated with FcBlock for 10 min at $4{ }^{\circ} \mathrm{C}$ and stained with PE-Cy7-anti-CD11c, APC-anti-CD45.2 and Brilliant-Violet-anti-CD45.1. Migrating CD11c high CD45.2 ${ }^{+}$ DCs were quantified by flow cytometry with a CyanTM ADP (Beckman Coulter) and analysed using FlowJo software (FlowJo Company, USA).

\section{In vitro transwell migration assay. BMDCs were washed and resuspended in} migration medium ( $1 \%$ BSA, $10 \%$ serum in phenol-free DMEM). $5 \times 10^{5}$ cells were seeded onto $8.0 \mu \mathrm{m}$ pore cell culture inserts in a 24 -well plate (Corning), and either control medium or medium supplemented with $10^{-6} \mathrm{M}$ chemoattractant (fMIFL, SDF-1 $\alpha$ or CCL21) was added to the bottom chamber to initiate migration. Cells were incubated at $37^{\circ} \mathrm{C}$ for $24 \mathrm{~h}$, after which inserts were removed and plates were placed on ice, $5 \mathrm{mM}$ EDTA was added to each well, and nuclei were stained with 2.5 $\mu \mathrm{M}$ DRAQ5 (ThermoFisher). The total number of migrated DRAQ5 ${ }^{+}$cells was quantified. \% Migration is defined as the total number of migrated cells in response to chemoattractant divided by the total number of migrated cells in response to control medium minus 1 times $100 \%$.
Phagocytosis. For flow cytometry-based phagocytosis assays, OVA-coated YGFluoresbrite beads were added at a 20:1 ratio in duplicate for the indicated times. Cells were washed in FACS buffer and stained with anti-CD11c-APC antibodies as described above, and the mean fluorescence intensity (MFI) quantified for CD11 $\mathrm{c}^{+}$ cells. MFIs were normalized to the mean WT $4 \mathrm{~h}$ phagocytosis value performed on the same day. For microscopy-based phagocytosis assays cells were matured with $\mathrm{CpG}$, exposed to 20:1 OVAb for $4 \mathrm{~h}$, washed, fixed in $4 \%$ paraformaldehyde/PBS, permeabilized and blocked in $0.1 \%$ triton-X/1\% BSA, stained with $0.6 \mu \mathrm{M}$ TRITCphalloidin and $10 \mu \mathrm{g} \mathrm{mL}^{-1}$ Hoechst 33342 and visualized using confocal microscopy.

Immunofluorescence. Immunostaining of BMDCs with anti-STIM1 was performed as described ${ }^{17}$ except that cells were fixed for $10 \mathrm{~min}$ in $2 \%$ paraformaldehyde(PFA)/PBS. Briefly, cells were permeabilized in $0.1 \%$ Triton-X for 5 min, washed $3 \times \mathrm{PBS}$, reduced with $0.1 \% \mathrm{NaBH}_{4} / \mathrm{PBS}$, blocked with Image-IT-FX (Thermo) for $20 \mathrm{~min}$, blocked with $0.5 \% \mathrm{BSA} / \mathrm{PBS}+\mathrm{FcBlock}$ for $30 \mathrm{~min}$, incubated overnight in primary antibody and for $1 \mathrm{~h}$ in secondary antibody in $0.5 \%$ BSA/PBS. For anti-IRAP immunostainings, cells were exposed to either 20:1 Alexa-488 OVAb or lysine-fixable FITC-dextran $\left(10,000 \mathrm{kDa}, 50 \mu \mathrm{g} \mathrm{m} \mathrm{m}^{-1}\right)$ for $15 \mathrm{~min}$ prior to fixation for 10 or $30 \mathrm{~min}$, respectively, in $4 \%$ (PFA/PBS). For OVAb stainings, cells were then blocked in $0.5 \% \mathrm{BSA} / 0.2 \%$ saponin/PBS+FcBlock for $30 \mathrm{~min}$ and primary and secondary antibodies applied as above in the same buffer. For dextran stainings, cells were permeabilized in $0.01 \%$ Triton-X/PBS for $3 \mathrm{~min}$, washed $3 \times$ in PBS, blocked in $0.5 \%$ BSA/PBS+FcBlock, and similarly incubated with primary and secondary antibodies.

$\mathbf{C a}^{2+}$ imaging. BMDCs and DC ${ }^{2114}$ cells seeded on Fluorodishes (WPI, USA) and, where indicated, matured as described above. Cells were loaded with $4 \mu \mathrm{M}$ Fura-2$\mathrm{AM}, 0.01 \%$ pluronic (ThermoFisher) in modified Ringer's $\mathrm{s}^{17}$ for $30 \mathrm{~min}$ at room temperature (RT). $340 / 380 \mathrm{~nm}$ excitation $510 \pm 40 \mathrm{~nm}$ emission ratiometric imaging was performed at $37^{\circ} \mathrm{C}$ in modified Ringer's where $\mathrm{Ca}^{2+}$-free solution contained $1 \mathrm{mM}$ EGTA instead of $2 \mathrm{mM} \mathrm{CaCl}$. Frames were acquired every $3 \mathrm{~s} . \mathrm{Ca}^{2+}$ microdomain/hotspot imaging was performed as described ${ }^{17}$ except cells were loaded with $4 \mu \mathrm{M}$ Fluo-8-AM (AAT Bioquest) for $30 \mathrm{~min}$ at $37^{\circ} \mathrm{C}, 30 \mathrm{~min}$ at RT and $2.5 \mu \mathrm{M}$ BAPTA-AM for the last $10 \mathrm{~min}$, in modified Ringer's containing 500 $\mu \mathrm{M}$ sulfinpyrazone. Simultaneous excitation at 488 and $543 \mathrm{~nm}$ and emission collection in two separate channels for green (Fluo8) and red (mCherry) was used. For quantification of periphagosomal $\mathrm{Ca}^{2+}$ hotspots, images were averaged over $6 \mathrm{~s}$ and captured between 20 and $30 \mathrm{~min}$ after the addition of OVAb. Where applicable Xestospongin $\mathrm{C}(1 \mu \mathrm{M})$ was applied $20 \mathrm{~min}$ after exposure of BMDCs to OVAb and hotspots imaged at $30 \mathrm{~min}$, while GSK7975A (a gift from Dr. Martin Lochner, University of Bern, $10 \mu \mathrm{M}$ ) was applied concurrently with OVAb and images taken after $30 \mathrm{~min}$.

Western blotting. Cells were washed with ice cold PBS and lysates prepared as described $^{17}$. Total protein was quantified using a BCA assay (ThermoFisher) according to the manufacturer's instructions. $50 \mu \mathrm{g}$ per lane were loaded for ORAI1 gels, while $30 \mu \mathrm{g}$ per lane were loaded for all others. 4-20\% Mini-Protean TGX Precast gels (BioRad) and iBlot PVDF kits (ThermoFisher) were used for SDS-PAGE and transfer respectively. All antibodies were diluted in 3\% milk/1\%Tween/TBS, primary antibodies were incubated overnight and secondary antibodies incubated for $1 \mathrm{~h}$. Membranes were visualized using Immobilon Western Chemiluminescent HRP Substrate (Millipore) and a PXi gel imaging system (Syngene, UK).

Electron microscopy. Classic transmission electron microscopy (TEM) and 3D Focused-ion beam scanning electron microscopy (FIB-SEM) were performed with the help of the Electron Microscopy Core Facility of the University of Geneva. TEM samples were prepared and imaged as described ${ }^{17}$. FIB-SEM was performed on a Helios NanoLab G3 microscope (FEI, Netherlands) with images acquired using the electron beam at $2 \mathrm{kV} / 0.2 \mathrm{nA}$, and milling sections with the ion beam at $30 \mathrm{kV} / 2.5 \mathrm{nA}$ every $10 \mathrm{~nm}$. Specimens were prepared using a protocol modified from $^{64}$. Cells were fixed in $2.5 \%$ glutaraldehyde, $2 \% \mathrm{PFA}$ in $2 \mathrm{mM} \mathrm{CaCl}_{2}$ in $0.15 \mathrm{M}$ cacodylate buffer ( $\mathrm{pH}$ 7.4) (Ca-Caco) for $3 \mathrm{~h}$ on ice and washed $5 \times$ in cold $\mathrm{Ca}$ Caco. Samples were incubated for $1 \mathrm{~h}$ on ice in $2 \% \mathrm{OsO}_{4}, 1.5 \% \mathrm{KFCN}$ in Ca-Caco and washed $5 \times$ in cold $\mathrm{ddH}_{2} \mathrm{O}$. Samples were then incubated in a freshly prepared and filtered $1 \%$ thiocarbohydrazide for 20 min at RT, washed $5 \times$ in $\mathrm{RT}^{2} \mathrm{ddH}_{2} \mathrm{O}$, incubated in $2 \% \mathrm{OsO}_{4}$ for $30 \mathrm{~min}$ at $\mathrm{RT}$ and then incubated in cold $1 \%$ uranyl acetate at $4{ }^{\circ} \mathrm{C}$ overnight. Samples were then washed $5 \times$ in $\mathrm{RT} \mathrm{ddH}_{2} \mathrm{O}$ and stained using Walton's lead aspartate method for $30 \mathrm{~min}$ at $60^{\circ} \mathrm{C}$, and rinsed $5 \times$ in RT $\mathrm{ddH}_{2} \mathrm{O}$. Samples were dehydrated in $2 \times 7$ min steps of cold $20,50,70,90,100$ and $100 \%$ anhydrous ethanol, then cold anhydrous acetone for $10 \mathrm{~min}$ and RT acetone for $10 \mathrm{~min}$. Samples were then embedded in hard epon resin by infiltrating with 25 , 50 and $75 \%$ hard epon/acetone at RT for $2 \mathrm{~h}$ each, then $100 \%$ hard epon overnight Fresh $100 \%$ hard epon was exchanged the next day incubated for $3 \mathrm{~h}$. Epon was once more exchanged before polymerization for $48 \mathrm{~h}$ at $60^{\circ} \mathrm{C}$. Cut and microtometrimmed blocks were mounted on aluminium pins using Pelco C100 cyanoacrylate glue and Pelco conductive silver paint (Ted Pella, USA). Samples were sputtercoated with gold for $20 \mathrm{~s}$ using a Q150T ES coater (Quorum Technologies, UK). 
pH measurements. Phagosomal pH was measured using FITC-coupled zymosan added at 20:1 ratio as described ${ }^{65}$ except that calibrations ( $\left.\mathrm{pH} 4.0-9.0\right)$ were performed using five different stacks per condition for each calibration buffer rather than a single time-lapse acquisition. pHrodo Red Zymosan Bioparticles (ThermoFisher) were washed $3 \times$ in sterile filtered $0.1 \mathrm{M} \mathrm{NaCO}_{3}$ buffer ( $\mathrm{pH} 8.0$ ), $10 \mu \mathrm{g}$ $\mathrm{mL}^{-1}$ Alexa-488-succinimidyl ester (SE) was added and particles were agitated for $4 \mathrm{~h}$ at RT. Unbound dye was removed by washing in $50,33 \%$, then $25 \%$ DMSO/ PBS and $3 \times$ in sterile PBS. Zymosan was then incubated in $20 \mathrm{mg} \mathrm{mL}^{-1}$ OVA overnight and washed $3 \times$ in PBS. Measurements, calibration and analysis were obtained using an identical protocol to FITC-coupled zymosan with the following exceptions: imaging was performed using alternate 555/590 and 488/530 nm Ex/ Em illumination. Zymosan were added to cells in complete medium and were washed in modified Ringer's after $30 \mathrm{~min}$, and imaged 30 and/or $90 \mathrm{~min}$ after addition. Where applicable, $0.2 \mathrm{nM}$ ConcA was added immediately before zymosan. Endosomal pH measurements were made as previously described ${ }^{66}$. Briefly, cells were loaded with with $1 \mathrm{mg} \mathrm{mL}^{-1}$ of FITC- and Alexa-647-labelled $40 \mathrm{kDa}$ dextrans (Thermo) for $10 \mathrm{~min}$ at $37^{\circ} \mathrm{C}$ and extensively washed with cold PBS/1\% BSA. Cells were then chased for different times and analysed by flow cytometry.

ROS measurements. Extracellular ROS were measured on cells seeded at 50,000 cells per well in black/clear bottom plates using the Amplex Red Ultra Hydrogen Peroxide/Peroxidase kit (ThermoFisher), according to the manufacturer's instructions. Intracellular ROS were measured on cells similarly plated. Dihydroethidium (DHE) $(30 \mu \mathrm{M})$ was loaded for $1 \mathrm{~h}$ in complete medium at $37^{\circ} \mathrm{C}$ before washing in modified Ringer's. For both assays fluorescence was measured every $2 \mathrm{~min}$ for $90 \mathrm{~min}$ at $37^{\circ} \mathrm{C}$ after addition of the indicated stimuli $(100 \mathrm{nM}$ PMA, 20:1 zymosan or 20:1 OVAb) using a SpectraMAX Paradigm plate reader, and is reported as the maximum fluorescence value normalized to the average baseline value over the first $6 \mathrm{~min}$. Intraphagosomal/intracellular ROS was measured on cells plated on Fluorodishes using OxyBurst/Alexa-568-coupled beads. $3.0 \mu \mathrm{m}$ Polybead Amino Microspheres (Polysciences) were washed in $3 \times$ in sterile filtered $0.1 \mathrm{M} \mathrm{NaCO}_{3}$ buffer (pH 8.0), $20 \mu \mathrm{g} \mathrm{mL}{ }^{-1}$ OxyBurst-H2DCFDA-SE (ThermoFisher) was added and particles were agitated for overnight at RT in tubes filled with nitrogen. Alexa-568-SE (ThermoFisher) was then added for $1 \mathrm{~h}$. Unbound dye was removed as above; beads were coated with OVA as above and beads were stored under nitrogen. OxyBurst/Alexa568-OVA beads were added to cells at 20:1 in complete medium, were washed in modified Ringer's after $30 \mathrm{~min}$, and imaged using alternate 555/590, 488/530 nm Ex/Em and brightfield illumination 30 and/or $90 \mathrm{~min}$ after addition. Where applicable cells were pre-incubated for $10 \mathrm{~min}$ with $10 \mu \mathrm{M}$ DPI prior to stimulus addition. As OxyBurst leaked from phagosomes into the cytosol, brightfield images were used to demarcate cell borders and the ratio of whole-cell OxyBurst to whole-cell Alexa-568 fluorescence minus the basal (external bead) ratio calculated as an index of intracellular/phagosomal ROS

Measurement of cytosol transfer. Measurement of cytosol transfer was performed as previously described ${ }^{16}$. Briefly, cells were loaded with $4 \mu \mathrm{M}$ CCF4-AM probe (a FRET-sensitive cytosolic substrate of $\beta$-lactamase, ThermoFisher) for $1 \mathrm{~h}$, washed with PBS and incubated at $37^{\circ} \mathrm{C}$ with $2 \mathrm{mg} \mathrm{mL}^{-1} \beta$-lactamase for different time points. Reactions were stopped with cold PBS and live, single, $\mathrm{CD} 11 \mathrm{c}^{+}$cells were analysed on a BD Biosciences Fortessa flow cytometer by monitoring the increase in $450 \mathrm{~nm}$ fluorescence and loss of $535 \mathrm{~nm}$ emission fluorescence resulting from CCF4 cleavage.

Proteolysis assays. Phagosomal proteolysis was measured using a protocol modified from ref. ${ }^{30}$. 3.0 $\mu \mathrm{m}$ Polybead Amino Microspheres (Polysciences) were labelled with Alexa-568-SE as described above. Labelled beads were then washed in $25 \mathrm{mM}$ sodium citrate, $25 \mathrm{mM}$ sodium phosphate buffer ( $\mathrm{pH}$ 5.0), and incubated with $20 \mathrm{mg} \mathrm{mL}^{-1} \mathrm{DQ}$-ovalbumin (DQ-OVA, ThermoFisher) overnight at $4^{\circ} \mathrm{C}$ on an end-over-end rotor, followed by three washes in sterile PBS (ThermoFisher). Cells were exposed to beads, imaged and analysed as described above for OxyBurst assays. Where applicable cells were pre-loaded for $30 \mathrm{~min}$ with $40 \mu \mathrm{M}$ BAPTA-AM, and washed prior to stimulus addition, and $0.2 \mathrm{nM}$ ConcA added just prior to beads.

Phagosomal OVA degradation assay. Phagosomal OVA degradation assay was performed as previously described ${ }^{66}$. Briefly, $3.0 \mu \mathrm{m}$ Polybead Amino Microspheres (Polysciences) were covalently coated with OVA $\left(0.2 \mathrm{mg} \mathrm{mL}^{-1}\right)$ according to the manufacturer's instructions. BMDCs were pulsed for $15 \mathrm{~min}$ at $37^{\circ} \mathrm{C}$ and chased for different times at $37^{\circ} \mathrm{C}$. Cells were lysed in $50 \mathrm{mM}$ Tris- $\mathrm{HCl}$ (pH 7.4) supplemented with $150 \mathrm{mM} \mathrm{NaCl}, 1 \mathrm{mM}$ DTT, $0.5 \% \mathrm{NP}-40$, and cocktail of protease inhibitors (Roche). For flow cytometry experiments, beads were stained with polyclonal anti-OVA (Sigma) and a secondary anti-rabbit Alexa488 (Thermo), and analysed on a BD Biosciences Fortessa flow cytometer.

Protease activity assay. Cathepsin/AEP protease activity assays were performed as previously described ${ }^{66}$ using $1 \mu \mathrm{g}$ of protein in lysis buffer $(50 \mathrm{mM}$ Tris $/ 150 \mathrm{mM}$ $\mathrm{NaCl} / 1 \% \mathrm{NP} 40$ ) on a Mithras LB 940 microplate reader by measuring the release of fluorescent N-Acetyl-Methyl-Coumarin substrate $(100 \mu \mathrm{M})$ in citrate buffer $(\mathrm{pH}$ $5.5)$ at $37^{\circ} \mathrm{C}$, detected using $380 / 440 \mathrm{~nm}$ excitation/emission. Leucyl aminopeptidase activity was measured as previously described ${ }^{67}$ in $50 \mathrm{mM}$ Tris buffer, $\mathrm{pH} 7.5 / 1 \mathrm{mM}$ DTT and $100 \mu \mathrm{M}$ substrate except that activity was measured on $3 \mu \mathrm{g}$ of whole-cell or purified phagosome lysates without anti-IRAP immunoprecipitation. Specific substrates for AEP (Z-Ala-Ala-Asn- NHMec), CatB/L (ZPhe-Arg-NHMec), CatS (Z-Val-Val-Arg-NHMec) and IRAP substrate (Leu-AMC) were purchased from Bachem.

Phagosome isolation for leucyl aminopeptidase activity. $1.5 \times 10^{7}$ BMDCs were exposed to $3 \times 10^{8}$ magnetic $3 \mu \mathrm{m}$ OVA-coated beads (Polysciences) for $15 \mathrm{~min}$ on ice. Warm medium was added and cells were incubated for $20 \mathrm{~min}$ at $37^{\circ} \mathrm{C}$. Cells were returned to ice and washed twice in cold PBS and once in cold IEB buffer containing protease inhibitor cocktail (PI, Sigma) (IEB: $10 \mathrm{mM}$ HEPES, $1 \mathrm{mM}$ EGTA, $25 \mathrm{mM} \mathrm{KCL}, 250 \mathrm{mM}$ sucrose). Cells were scraped, centrifuged, and washed once in IEB/PI and counted. Cells were then centrifuged and resuspended in IEB/ $\mathrm{PI}$ at a concentration of $4 \times 10^{6}$ cells $\mathrm{mL}^{-1}$, and passed 20x through a $20 \mu \mathrm{m}$ clearance ball-bearing homogenizer. Homogenates were then transferred to a magnet and washed once in IEB/PI, once in IEB/PI $+4 \mathrm{mM} \mathrm{MgCl}_{2}+10 \mathrm{mM} \mathrm{Na}-$ ATP, and were then incubated on an end-over-end rotor for $15 \mathrm{~min}$ at $4{ }^{\circ} \mathrm{C}$. Homogenates were washed one final time on a magnet and resuspended in lysis buffer (50 mM Tris; $150 \mathrm{mM} \mathrm{NaCl}$; $1 \%$ NP40) without protease inhibitors. Lysates were incubated on ice for $30 \mathrm{~min}$ prior to quantification using a BCA kit (ThermoFisher).

Phago-lysosome and phago-endosome fusion assay. Phago-lysosome fusion was measured using a protocol modified from ${ }^{30} .3 .0 \mu \mathrm{m}$ Polybead Amino Microspheres (Polysciences) were labelled with Alexa-488-SE and coated with OVA as described above. Twenty four hours prior to the assay cells were pulse labelled with $20 \mu \mathrm{g} \mathrm{mL}^{-1}$ Alexa-594-HA (ThermoFisher) in complete medium for $3 \mathrm{~h}$, washed, and followed by a chase period of $24 \mathrm{~h}$ to allow the dye to accumulate in lysosomes. Alexa488-OVA beads were added to cells at 20:1 in complete medium, were washed in modified Ringer's after 30 min, and imaged using alternate 555/590, 488/ 530, 488/590 nm Ex/Em and brightfield illumination 30 and/or 90 min after addition. When applicable cells were pre-loaded for $30 \mathrm{~min}$ with $40 \mu \mathrm{M}$ BAPTA$\mathrm{AM}$, and washed prior to stimulus addition and $0.2 \mathrm{nM}$ ConcA added just prior to beads. The P-L fusion index is computed as the ratio of the FRET (488/590)/Alexa488 (488/530) signal minus the basal (external bead) FRET signal, normalized to the total average cellular Alexa-594-HA fluorescence. Phago-endosome fusion assays were performed in a similar manner except that $1 \mu \mathrm{gL}^{-1}$ Alexa-594dextran of 10,000 MW (ThermoFisher) was added $15 \mathrm{~min}$ prior to beads, cells were washed $3 \times$ in complete medium prior to Alexa488-OVA bead addition and external beads were washed in modified Ringer's after $15 \mathrm{~min}$. Where applicable, GSK7975A $(10 \mu \mathrm{M})$ was added concurrently with beads.

Image analysis and statistics. All fluorescence image analyses were performed using ImageJ software $(\mathrm{NIH})$ on maximum projections of at least $5 \times 15 \mu \mathrm{m}$ $z$-stacks per condition, except for IRAP quantification which were performed on a single confocal plane from each stack, and which were taken with a 100× objective. Semi-automated segmentation based on a single visually determined threshold for the $\mathrm{pH} / \mathrm{ROS} /$ proteolysis insensitive wavelength did not fully separate all closely clustered-phagosomes particles, and thus single-phagosome measurements include an estimated $20-30 \%$ phagosome clusters. External (non-cell-associated) zymosan/ beads were used to estimate time 0 ratio values and then eliminated manually from the analysis. For IRAP quantification, the total periphagosomal IRAP signal (obtained from the bead segmentation mask, and measured in a single plane that captured most phagosomes within a stack at their mid-section) was normalized to the total IRAP cellular fluorescence, calculated from sum projections of the same stack. Alignment, segmentation and 3D reconstruction of FIB-SEM images was performed using Amira software (FEI). Quantification of ER-Ph MCS was conducted in single-blind and performed manually on $10 \mathrm{~nm}$ slices, at 30 slice intervals to avoid double counting of MCS. MCS were defined as areas where the ER was $<30 \mathrm{~nm}$ away from the phagosomal membrane. Phagosome cross-sections smaller than $1.5 \mu \mathrm{m}$ in diameter were excluded from the analysis to avoid any undersampling bias of phagosomal membranes. MCS frequency was defined as the number of MCS (also called ER junctions) detected divided by the total number of phagosomes analysed, multiplied by 100 to obtain the average number of ER junctions per 100 phagosomes. Animal studies were not conducted blind, but at least $N=4$ animals were tested for each condition, and, within each genotype, were randomly assigned to control and experimental groups. All statistical analyses were performed using Prism 6.0 software (GraphPad). Pairwise comparisons were made using a Mann-Whitney test for experiments based on phagocytosis, as phagocytic events do not follow a Gaussian distribution. A two-sided Student's $t$-test was used for all other pairwise comparisons, and, where $F$-test showed significantly difference variances, Welch's correction was applied. A Two-way ANOVA with Sidak's multiple comparisons test was used for repeated measures. $N=$ number of independent experiments, at least three independent experiments were performed for all conditions.

Data availability. The data that support the findings of this study are available from the corresponding author upon reasonable request. 
Received: 7 October 2016 Accepted: 2 October 2017

Published online: 24 November 2017

\section{References}

1. Ackerman, A. L. \& Cresswell, P. Cellular mechanisms governing crosspresentation of exogenous antigens. Nat. Immunol. 5, 678-684 (2004).

2. Manolova, V. et al. Nanoparticles target distinct dendritic cell populations according to their size. Eur. J. Immunol. 38, 1404-1413 (2008).

3. Gagnon, E. et al. Endoplasmic reticulum-mediated phagocytosis is a mechanism of entry into macrophages. Cell 110, 119-131 (2002).

4. Campbell-Valois, F. X. et al. Quantitative proteomics reveals that only a subset of the endoplasmic reticulum contributes to the phagosome. Mol. Cell. Proteom. 11, M111 016378 (2012).

5. Guermonprez, P. et al. ER-phagosome fusion defines an MHC class I crosspresentation compartment in dendritic cells. Nature 425, 397-402 (2003).

6. Touret, N., Paroutis, P. \& Grinstein, S. The nature of the phagosomal membrane: endoplasmic reticulum versus plasmalemma. J. Leukoc. Biol. 77, 878-885 (2005).

7. Zehner, M. et al. The translocon protein Sec61 mediates antigen transport from endosomes in the cytosol for cross-presentation to CD8(+) T cells. Immunity 42, 850-863 (2015).

8. Nair-Gupta, P. et al. TLR signals induce phagosomal MHC-I delivery from the endosomal recycling compartment to allow cross-presentation. Cell 158, 506-521 (2014).

9. Demaurex, N. \& Nunes, P. The role of STIM and ORAI proteins in phagocytic immune cells. Am. J. Physiol. Cell Physiol. 310, C496-C508 (2016).

10. Hogan, P. G. \& Rao, A. Store-operated calcium entry: mechanisms and modulation. Biochem. Biophys. Res. Commun. 460, 40-49 (2015).

11. Shen, W. W. \& Demaurex, N. Morphological and functional aspects of STIM1dependent assembly and disassembly of store-operated calcium entry complexes. Biochem. Soc. Trans. 40, 112-118 (2012).

12. Hsu, S. et al. Fundamental Ca2+ signaling mechanisms in mouse dendritic cells: CRAC is the major Ca2+ entry pathway. J. Immunol. 166, 6126-6133 (2001).

13. Bandyopadhyay, B. C., Pingle, S. C. \& Ahern, G. P. Store-operated Ca(2)+ signaling in dendritic cells occurs independently of STIM1. J. Leukoc. Biol. 89, 57-62 (2011).

14. Felix, R. et al. The Orai-1 and STIM-1 complex controls human dendritic cell maturation. PLOS ONE 8, e61595 (2013).

15. Vaeth, M. et al. Ca2+ Signaling but not store-operated $\mathrm{Ca} 2+$ entry is required for the function of macrophages and dendritic cells. J. Immunol. 195, 1202-1217 (2015).

16. Cebrian, I. et al. Sec22b regulates phagosomal maturation and antigen crosspresentation by dendritic cells. Cell 147, 1355-1368 (2011).

17. Nunes, P. et al. STIM1 juxtaposes ER to phagosomes, generating $\mathrm{Ca}(2)(+)$ hotspots that boost phagocytosis. Curr. Biol. 22, 1990-1997 (2012).

18. Steiner, Q. G. et al. In vivo transformation of mouse conventional CD8alpha + dendritic cells leads to progressive multisystem histiocytosis. Blood 111, 2073-2082 (2008)

19. Kabashima, K. et al. CXCL12-CXCR4 engagement is required for migration of cutaneous dendritic cells. Am. J. Pathol. 171, 1249-1257 (2007).

20. Shao, Z., Gaurav, R. \& Agrawal, D. K. Intermediate-conductance calciumactivated potassium channel KCa3.1 and chloride channel modulate chemokine ligand (CCL19/CCL21)-induced migration of dendritic cells. Transl. Res. 166, 89-102 (2015).

21. Scandella, E. et al. CCL19/CCL21-triggered signal transduction and migration of dendritic cells requires prostaglandin E2. Blood 103, 1595-1601 (2004).

22. Nunes, P. \& Demaurex, N. The role of calcium signaling in phagocytosis. J. Leukoc. Biol. 88, 57-68 (2010).

23. Merad, M., Sathe, P., Helft, J., Miller, J. \& Mortha, A. The dendritic cell lineage: ontogeny and function of dendritic cells and their subsets in the steady state and the inflamed setting. Annu. Rev. Immunol. 31, 563-604 (2013).

24. Steinckwich, N., Schenten, V., Melchior, C., Brechard, S. \& Tschirhart, E. J. An essential role of STIM1, Orai1, and S100A8-A9 proteins for Ca2+ signaling and FcgammaR-mediated phagosomal oxidative activity. J. Immunol. 186, 2182-2191 (2011).

25. Zhang, H. et al. STIM1 calcium sensor is required for activation of the phagocyte oxidase during inflammation and host defense. Blood 123, 2238-2249 (2014)

26. Kotsias, F., Hoffmann, E., Amigorena, S. \& Savina, A. Reactive oxygen species production in the phagosome: impact on antigen presentation in dendritic cells. Antioxid. Redox Signal. 18, 714-729 (2013).

27. Yates, R. M. \& Russell, D. G. Real-time spectrofluorometric assays for the lumenal environment of the maturing phagosome. Methods Mol. Biol. 445, 311-325 (2008).
28. Savina, A., Vargas, P., Guermonprez, P., Lennon, A. M. \& Amigorena, S. Measuring $\mathrm{pH}$, ROS production, maturation, and degradation in dendritic cell phagosomes using cytofluorometry-based assays. Methods Mol. Biol. 595, 383-402 (2010).

29. Ackerman, A. L., Kyritsis, C., Tampé, R. \& Cresswell, P. Access of soluble antigens to the endoplasmic reticulum can explain cross-presentation by dendritic cells. Nat. Immunol. 6, 107-113 (2005).

30. Podinovskaia, M. et al. Dynamic quantitative assays of phagosomal function. Curr. Protoc. Immunol. 102, Unit 14.34 (2013).

31. Blum, J. S., Wearsch, P. A. \& Cresswell, P. Pathways of antigen processing. Annu. Rev. Immunol. 31, 443-473 (2013).

32. Saveanu, L. et al. IRAP identifies an endosomal compartment required for MHC class I cross-presentation. Science 325, 213-217 (2009).

33. Rao, S., Liu, X., Freedman, B. D. \& Behrens, E. M. Spleen tyrosine kinase (Syk)dependent calcium signals mediate efficient CpG-induced exocytosis of tumor necrosis factor alpha (TNFalpha) in innate immune cells. J. Biol. Chem. 288, 12448-12458 (2013).

34. Kremlitzka, M., Macsik-Valent, B. \& Erdei, A. Syk is indispensable for CpGinduced activation and differentiation of human B cells. Cell. Mol. Life Sci. 72, 2223-2236 (2015).

35. Nunes, P., Demaurex, N. \& Dinauer, M. C. Regulation of the NADPH oxidase and associated ion fluxes during phagocytosis. Traffic 14, 1118-1131 (2013).

36. Rybicka, J. M., Balce, D. R., Chaudhuri, S., Allan, E. R. \& Yates, R. M. Phagosomal proteolysis in dendritic cells is modulated by NADPH oxidase in a pH-independent manner. EMBO J. 31, 932-944 (2012).

37. Savina, A. et al. The small GTPase Rac2 controls phagosomal alkalinization and antigen crosspresentation selectively in CD8(+) dendritic cells. Immunity 30, 544-555 (2009).

38. Savina, A. et al. NOX2 controls phagosomal $\mathrm{pH}$ to regulate antigen processing during crosspresentation by dendritic cells. Cell 126, 205-218 (2006).

39. El Chemaly, A., Nunes, P., Jimaja, W., Castelbou, C. \& Demaurex, N. Hv1 proton channels differentially regulate the $\mathrm{pH}$ of neutrophil and macrophage phagosomes by sustaining the production of phagosomal ROS that inhibit the delivery of vacuolar ATPases. J. Leukoc. Biol. 95, 827-839 (2014).

40. Jankowski, A., Scott, C. C. \& Grinstein, S. Determinants of the phagosomal pH in neutrophils. J. Biol. Chem. 277, 6059-6066 (2002).

41. Tlili, A., Dupre-Crochet, S., Erard, M. \& Nusse, O. Kinetic analysis of phagosomal production of reactive oxygen species. Free Radic. Biol. Med. 50, 438-447 (2011).

42. Tsujikawa, H. et al. Identification of key amino acid residues responsible for internal and external pH sensitivity of Orail/STIM1 channels. Sci. Rep. 5, 16747 (2015).

43. Alloatti, A. et al. Toll-like receptor 4 engagement on dendritic cells restrains phago-lysosome fusion and promotes cross-presentation of antigens. Immunity 43, 1087-1100 (2015).

44. Singh, R. \& Cresswell, P. Defective cross-presentation of viral antigens in GILTfree mice. Science 328, 1394-1398 (2010).

45. Kurotaki, T. et al. Efficient cross-presentation by heat shock protein 90 -peptide complex-loaded dendritic cells via an endosomal pathway. J. Immunol. 179, 1803-1813 (2007).

46. Flinsenberg, T. W. et al. Fcgamma receptor antigen targeting potentiates crosspresentation by human blood and lymphoid tissue BDCA-3+ dendritic cells. Blood 120, 5163-5172 (2012).

47. Accapezzato, D. et al. Chloroquine enhances human CD8+ T cell responses against soluble antigens in vivo. J. Exp. Med. 202, 817-828 (2005).

48. Jancic, C. et al. Rab27a regulates phagosomal $\mathrm{pH}$ and NADPH oxidase recruitment to dendritic cell phagosomes. Nat. Cell Biol. 9, 367-378 (2007).

49 Maschalidi S. et al. UNC93B1 interacts with the calcium sensor STIM1 for efficient antigen cross-presentation in dendritic cells. Nat. Commun. doi: 10.1038/s41467-017-01601-5 (2017).

50. Oliveira, C. C. \& van Hall, T. Importance of TAP-independent processing pathways. Mol. Immunol. 55, 113-116 (2013).

51. Kaufmann, U. et al. Selective ORAI1 inhibition ameliorates autoimmune central nervous system inflammation by suppressing effector but not regulatory T cell function. J. Immunol. 196, 573-585 (2016).

52. Endo, Y. et al. Dominant mutations in ORAI1 cause tubular aggregate myopathy with hypocalcemia via constitutive activation of store-operated $\mathrm{Ca}(2)$ (+) channels. Hum. Mol. Genet. 24, 637-648 (2015).

53. Darbellay, B., Barnes, L., Boehncke, W. H., Saurat, J. H. \& Kaya, G. Reversal of murine epidermal atrophy by topical modulation of calcium signaling. J. Investig. Dermatol. 134, 1599-1608 (2014).

54. Vandenberghe, M. et al. ORAI1 calcium channel orchestrates skin homeostasis. Proc. Natl Acad. Sci. USA 110, E4839-E4848 (2013).

55. Moccia, F. et al. Targeting Stim and Orai proteins as an alternative approach in anticancer therapy. Curr. Med. Chem. 23, 3450-3480 (2016).

56. Olson, D. K., Fröhlich, F., Farese, R. V. \& Walther, T. C. Taming the sphinx: Mechanisms of cellular sphingolipid homeostasis. Biochim. Biophys. Acta 1861, 784-792 (2016). 
57. Luik, R. M., Wu, M. M., Buchanan, J. \& Lewis, R. S. The elementary unit of store-operated $\mathrm{Ca} 2+$ entry: local activation of CRAC channels by STIM1 at ERplasma membrane junctions. J. Cell Biol. 174, 815-825 (2006).

58. Suter, D. M. et al. Rapid generation of stable transgenic embryonic stem cell lines using modular lentivectors. Stem Cells 24, 615-623 (2006).

59. Clausen, B. E., Burkhardt, C., Reith, W., Renkawitz, R. \& Forster, I. Conditional gene targeting in macrophages and granulocytes using LysMcre mice. Transgenic Res. 8, 265-277 (1999).

60. Oh-Hora, M. et al. Dual functions for the endoplasmic reticulum calcium sensors STIM1 and STIM2 in T cell activation and tolerance. Nat. Immunol. 9, 432-443 (2008).

61. Hogquist, K. A. et al. T cell receptor antagonist peptides induce positive selection. Cell 76, 17-27 (1994).

62. Shen, F. W. et al. Cloning of Ly-5 cDNA. Proc. Natl Acad. Sci. USA 82, 7360-7363 (1985)

63. Schaefer, B. C., Schaefer, M. L., Kappler, J. W., Marrack, P. \& Kedl, R. M. Observation of antigen-dependent CD8+ T-cell/ dendritic cell interactions in vivo. Cell. Immunol. 214, 110-122 (2001).

64. Deerinck, T. J., Bushong, E., Thor, A. \& Ellisman, M. H. NCMIR methods for 3D EM: a new protocol for preparation of biological specimens for serial block face scanning electron microscopy. National Center for Microscopy and Imaging Research https://www.ncmir.ucsd.edu/sbem-protocol/ (2010).

65. Nunes P., Guido D. \& Demaurex N. Measuring phagosome ph by ratiometric fluorescence microscopy. J. Vis. Exp. 106, e53402 (2015).

66. Sepulveda, F. E. et al. Critical role for asparagine endopeptidase in endocytic Toll-like receptor signaling in dendritic cells. Immunity 31, 737-748 (2009).

67. Babdor, J. et al. IRAP+ endosomes restrict TLR9 activation and signaling. Nat. Immunol. 18, 509-518 (2017).

\section{Acknowledgements}

We are grateful to the bioimaging core facility and the electron microscopy core facility (Geneva Medical Centre) and to Drs. Maud Frieden, Claes Wollheim and Isabelle Dunand-Sauthier for their valuable advice as well as to Nina Criado-Santos and Christian Vesin for technical assistance with immunostainings and animal experiments, respectively. This work was funded by the Swiss National Foundation [grant number 31003A149566 and CRSII3_ 160782 (to N.D.)]; COST Action BM1406, and a Young Investigator Subsidy from The Sir Jules Thorn Overseas Charitable Trust (to P.N.-H.).

\section{Author contributions}

P.N.-H. designed the study, designed and performed experiments, analysed and interpreted data, and wrote the manuscript. N.D. supervised study design, analysed and interpreted data, and edited the manuscript. B.M., S.M., C.L., S.H., designed and performed experiments, analysed and interpreted data. D.Me. and D.Ma. supervised and contributed research tools. D.Ma. also helped with the study design and data interpretation. S.B., D.G., and F.B. performed experiments and analysed data. C.C., E.Y.B., N.P. performed experiments.

\section{Additional information}

Supplementary Information accompanies this paper at doi:10.1038/s41467-017-01600-6.

Competing interests: The authors have no competing financial interests.

Reprints and permission information is available online at http://npg.nature.com/ reprintsandpermissions/

Publisher's note: Springer Nature remains neutral with regard to jurisdictional claims in published maps and institutional affiliations.

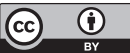

Open Access This article is licensed under a Creative Commons Attribution 4.0 International License, which permits use, sharing, adaptation, distribution and reproduction in any medium or format, as long as you give appropriate credit to the original author(s) and the source, provide a link to the Creative Commons license, and indicate if changes were made. The images or other third party material in this article are included in the article's Creative Commons license, unless indicated otherwise in a credit line to the material. If material is not included in the article's Creative Commons license and your intended use is not permitted by statutory regulation or exceeds the permitted use, you will need to obtain permission directly from the copyright holder. To view a copy of this license, visit http://creativecommons.org/ licenses/by/4.0/.

(C) The Author(s) 2017 